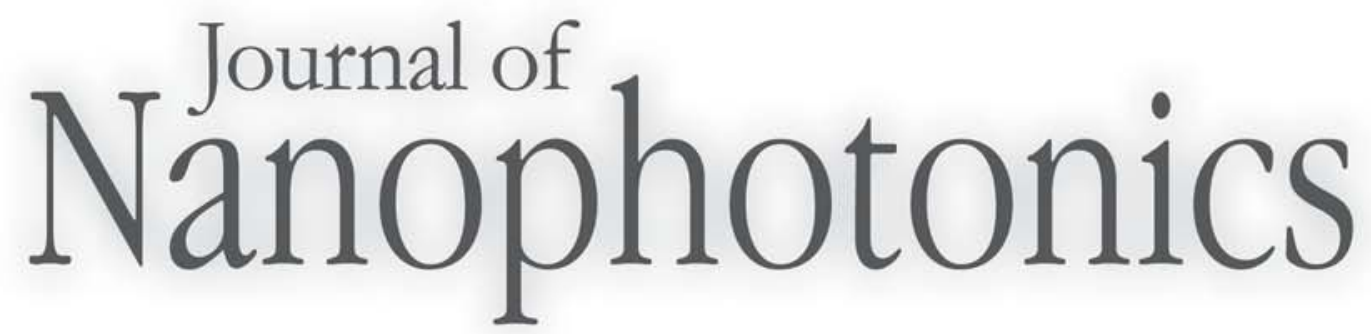

SPIEDigitalLibrary.org/jnp

\title{
Terahertz processes in carbon nanotubes
}

Konstantin G. Batrakov

Oleg V. Kibis

Polina P. Kuzhir

Marcelo Rosenau da Costa

Mikhail E. Portnoi 


\title{
Terahertz processes in carbon nanotubes
}

\section{Konstantin G. Batrakov, ${ }^{\text {a }}$ Oleg V. Kibis, ${ }^{\text {b }}$ Polina P. Kuzhir, ${ }^{\mathrm{a}}$ Marcelo Rosenau da Costa, ${ }^{\mathrm{c}}$ and Mikhail E. Portnoi ${ }^{\mathrm{d}}$}

\author{
a Belarus State University, Institute for Nuclear Problems, Bobruyskaya St. 11, 220030 Minsk, \\ Belarus \\ kgbatrakov@gmail.com \\ b Novosibirsk State Technical University, Department of Applied and Theoretical Physics, \\ Novosibirsk 630092, Russia \\ oleg.kibis@nstu.ru \\ ${ }^{\mathrm{c}}$ University of Brasília, International Center for Condensed Matter Physics, 70904-970 \\ Brasília DF, Brazil \\ rosenau@unb.br \\ d University of Exeter, School of Physics, Stocker Road, Exeter EX4 4QL, United Kingdom \\ m.e.portnoi@exeter.ac.uk
}

\begin{abstract}
We investigated several proposals utilizing the unique electronic properties of carbon nanotubes (CNTs) for a broad range of applications to THz optoelectronics, including $\mathrm{THz}$ generation by Čerenkov-type emitters based on carbon nanotubes and by hot electrons in quasimetallic nanotubes, frequency multiplication in chiral-nanotube-based superlattices controlled by a transverse electric field, and $\mathrm{THz}$ radiation detection and emission by armchair nanotubes in a strong magnetic field. Dispersion equations of the electron beam instability and the threshold conditions of the stimulated emission have been derived and analyzed, demonstrating realizability of the nanotube-based nanoFEL at realistic parameters of nanotubes and electronic beams.
\end{abstract}

Keywords: carbon nanotubes, terahertz radiation

\section{INTRODUCTION}

Creating a compact reliable source of terahertz $(\mathrm{THz})$ radiation is one of the most challenging problems in contemporary applied physics [1]. This great interest in the $\mathrm{THz}$ frequency range is fueled by the fact that $\mathrm{THz}$ frequencies characterize different important physical processes (rotation of small molecules, collective vibration modes of proteins, absorption in polar liquids, typical superconducting energy gaps, oscillations in gaseous and solid-state plasmas, etc). Reliable $\mathrm{THz}$ devices are required for air pollution monitoring, poison gas sensing, DNA manipulation, gene diagnostics, and other applications. Despite the fact that $\mathrm{THz}$ technology is at the boundaries of microwave and photonic technology, it is quite underdeveloped compared with the achievements in microwave or photonics. There are very few commercially available instruments for the $\mathrm{THz}$ frequency region and very often they lack the precision required for performing accurate measurements. There are also no miniaturized and low-cost THz sources. One of the latest trends is to use single-wall carbon nanotubes (SWNTs) - cylindrical molecules with nanometer diameter and micrometer length [2-4] — as building blocks of novel THz devices [5-7]. In this paper we summarize and discuss several schemes to utilize the physical properties of carbon nanotubes (CNTs) for generation and detection of THz radiation. 


\section{CARBON NANOTUBES AS ČERENKOV-TYPE TERAHERTZ EMITTERS}

Recently, the idea using the kinetic energy of CNT-guided electron beam for stimulated emission of electromagnetic waves in optical and terahertz ranges has been proposed [8-13]. In this section we present a consistent theory of the effect. There is a wide family of devices utilizing the interaction of electron beams with electromagnetic waves to produce electromagnetic radiation. Started by the invention of klystrons [14], this family embraces such well-known systems as traveling wave tubes (TWT) and backward wave oscillators (BWO) [15], free electron lasers (FEL) [16-19], etc. In such systems, synchronous motion of electrons and electromagnetic wave modulates the electron beam and coherent radiation is produced by electron bunches. The radiation frequency is smoothly tunable due to its dependence on the electron beam energy. Therefore, such types of emitters can operate in a wide spectral range from microwave and infrared frequencies to vacuum ultraviolet (e.g., VUV-FEL at DESY). Several projects aimed at lasing in hard X-ray range have started [20,21].The synchronization of moving electrons and electromagnetic wave is attained either by slowing down the electromagnetic wave (Čerenkov, Smith-Purcell [22] and quasi-Čerenkov [23] radiation mechanisms) or by applying an external magnetic f eld, which is uniform in gyrotrons [24,25] and spatially periodical in undulators [18]. The oscillator-type mechanism [26] can be also realized for electrons with discrete spectrum of transverse motion (for example, for electron channeling in crystals).

The Čerenkov radiation is governed by the synchronization condition $\omega-\mathbf{k u}=0$, where $\mathbf{k}$ is the wavevector and $\mathbf{u}$ is the charged particle (electron) velocity. In systems with external f elds the synchronism condition is transformed to $\omega-\mathbf{k u}-\Omega=0$, with $\Omega$ being the electron oscillation frequency. In the oscillator regime, $\Omega$ is the transition frequency between electron levels [27]. To achieve coherent generation in the devices described, a high vacuum must be maintained in the region of the electron beam [28]. Otherwise, collisions of electrons with atoms move electrons out of the synchronism and, consequently, lasing is not reached. From this point of view CNTs are unique objects since they exhibit ballistic electrical conduction at room temperature, with mean free paths of the order of microns and even tens of microns [29-31]. Therefore, electrons can emit coherently from the whole CNT length which is typically 1-10 $\mu \mathrm{m}$. In addition, single- and multi-wall carbon nanotubes can carry a high current density of the order of $10^{9}-10^{10} \mathrm{~A} / \mathrm{cm}^{2}$, see e.g. Refs. [32-34]. Lastly, metallic CNTs exhibit a strong slowing down of surface electromagnetic waves (as large as 50-100 times) $[35,36]$. Thus, a combination of three CNT key properties:

(i) ballisticity of the electron flow over typical CNT length,

(ii) extremely high current-carrying capacity and

(iii) strong slowing down of surface electromagnetic waves, makes them suitable candidates for the development of nano-sized Chernekov-type emitters nano-TWT, nano-BWO and nano-FEL.

\subsection{Self-consistent equation of motion for electromagnetic wave and electron beam}

Nanotubes - quasi-one-dimensional carbon macromolecules - are obtained by rolling a graphene layer into a cylinder. The transformation can be performed in different manners classif ed by the dual index $\left(n_{1}, n_{2}\right)$. The two integers $n_{1}$ and $n_{2}$ represent the vector characterizing the different ways of rolling, with $n_{1}=0$ for zigzag CNTs, $n_{1}=n_{2}$ for armchair CNTs, and $0<n_{1} \neq n_{2}$ for chiral CNTs. A nanotube can manifest either metallic or semiconductor properties, depending on its radius $R_{\mathrm{cn}}$ and how it is rolled. This correlation arises from the transverse quantization of charge carrier motion and is due to the quasi-one-dimensional topology of CNTs $[4,37]$.

Consider an electron beam moving in an isolated single-wall carbon nanotube oriented along the $z$-axis. The electron beam can be injected into the nanotube from the outside by an external 
source or they can be produced by applying voltage to some section of the nanotube. Accelerated by the voltage, electrons are injected into the working region. Independently on the origin of electrons, their motion in this region is assumed to be ballistic.

As was mentioned above, there is a certain analogy between a CNT guiding electron beam and macroscopic vacuum electron devices. The main (and obvious) distinction is the small cross-sectional radius of CNTs compared to their macroscopic analogs. In CNTs the spatial quantization of the electron motion comes into play and, therefore, classical models for electron beam become inapplicable. The electron motion in CNTs is governed by quantum-mechanical equations. In this paper we shall consider the lasing effect when the generated $\mathrm{f}$ eld is rather large, i.e. the condition

$$
E \gg \sqrt{\hbar c}\left(\frac{\omega}{c}\right)^{2}
$$

is fulfilled [38]. Further, from this place to the section 4, we set $\hbar=1$. In the case (1) the electromagnetic wave has a classical character and is described by the classical wave equation:

$$
\nabla \nabla \cdot \mathbf{E}(\mathbf{r}, \omega)-\Delta \mathbf{E}(\mathbf{r}, \omega)=\frac{4 \pi i \omega}{c^{2}} \mathbf{j}(\mathbf{r}, \omega)
$$

If condition (1) does not hold, the number of photons per quantum level becomes too small to apply a classical approach and the electromagnetic f eld must be considered within the quantum electrodynamics. Quantum-electrodynamical considerations are important in the initial stage of instability development, when a few photons participate in the process. We leave this stage for further analysis focusing on the stage of highly developed instability. Thus, in our model the electron motion is governed by the Schrödinger equation while the electromagnetic field is described by classical Maxwell equations. In the right-hand part of the feld equation (2) the quantity $\mathbf{j}(\mathbf{r}, \omega)$ is the current density averaged over the quantum states of the electron beam.

The current density in the working region is defined by the well-known equation [39]:

$$
\begin{aligned}
\mathbf{j}(\mathbf{r}, t) & =\frac{e}{2 m_{e}}\left\{\psi^{*}(\mathbf{r}, t) \hat{\mathbf{p}} \psi(\mathbf{r}, t)-\left(\hat{\mathbf{p}} \psi^{*}(\mathbf{r}, t)\right) \psi(\mathbf{r}, t)\right\} \\
& -\frac{e^{2}}{m_{e} c}|\psi(\mathbf{r}, t)|^{2} \mathbf{A}(\mathbf{r}, t) .
\end{aligned}
$$

Here $\hat{\mathbf{p}}=-i \hbar \partial /(\partial \mathbf{r})$ is the momentum operator and $\mathbf{A}(\mathbf{r}, t)$ is the vector potential of the electromagnetic field, $\psi(\mathbf{r}, t)$ is electron wave function. In what follows we neglect the Fermi law for the electron statistics. This is possible because the number of excited electrons per quantum level is found to be small, even at superior current densities reachable in CNTs [3234]. Indeed, the number of levels in the interaction volume $V$ is estimated as $\sim V p^{3} /(2 \pi \hbar)^{3}$, where $p$ is a typical value of the quasi-momentum of electrons in the beam. The number of electrons in this volume is $\sim n_{e} V$, where $n_{e}$ is the electron density. Then, the number of excited electrons per level is given by $\eta_{e}=(2 \pi \hbar)^{3} n_{e} / p^{3}$. At a current density of $10^{8}$ to $10^{10}$ $\mathrm{A} / \mathrm{cm}^{2}$ and an excitation energy of the order of several electronvolts, we find $\eta_{e} \sim 10^{-5}-10^{-3}$. Therefore, the exchange interaction between electrons in the beam can be neglected.

Let $\psi(\mathbf{r}, t=0)=\psi_{n}(\mathbf{r})$ be the eigenfunction of an electron not interacting with the electromagnetic wave and moving along the CNT. When the interaction is switched on the wavefunction is represented by the expansion

$$
\psi(\mathbf{r}, t)=\sum_{l} a_{l}(t) \exp \left(-i \varepsilon_{l} t\right) \psi_{l}(\mathbf{r})
$$

over a complete set of the unperturbed eigenfunctions $\psi_{l}(\mathbf{r})$ with corresponding energy eigenvalues $\varepsilon_{l}$. For further convenience, we rewrite the coefficients $a_{l}(t)$ as $a_{l}(t)=\delta_{l n}+\delta a_{l}^{(n)}(t)$, 
where $\delta_{l n}$ is the Kronecker symbol. The corrections $\delta a_{l}^{(n)}(t)$ are due to the electron-electromagnetic field interaction. Taking into account axial periodicity of the nanotube potential, the wavefunctions $\psi_{l}(\mathbf{r})$ can be written in accordance with Bloch theorem as

$$
\psi_{l}(\mathbf{r})=\exp \left\{i p_{l} z\right\} \sum_{\tau} b_{l \tau} \exp \{i \tau z\} u_{l \tau}\left(\mathbf{r}_{\perp}\right) .
$$

Here $p_{l}$ is the axial projection of the quasi-momentum of $l$-th state, $b_{l \tau}$ are constant coefficients, $\tau=2 \pi q / a$ are the reciprocal lattice constants, $a$ is the CNT spatial period in the axial direction, $u_{l \tau}\left(\mathbf{r}_{\perp}\right)$ are functions dependent only on transverse coordinates and $q$ are integers. The term $\sum_{\tau} b_{l \tau} \exp \{i \tau z\} u_{l \tau}\left(\mathbf{r}_{\perp}\right)$ is periodic in the $z$ direction.

In the linear approximation, the contribution to the electron current originating from the electron-electromagnetic field interaction is described by the equation:

$$
\begin{aligned}
\delta \mathbf{j}_{n}(\mathbf{r}, t) & =\frac{e}{2 m_{e}} \sum_{l}\left\{\delta a_{l}^{(n) *}(t) \exp \left[i\left(\varepsilon_{l}-\varepsilon_{n}\right) t\right]\left[\psi_{l}^{*}(\mathbf{r}) \hat{\mathbf{p}} \psi_{n}(\mathbf{r})-\left(\hat{\mathbf{p}} \psi_{l}^{*}(\mathbf{r})\right) \psi_{n}(\mathbf{r})\right]\right. \\
& \left.+\delta a_{l}^{(n)}(t) \exp \left[-i\left(\varepsilon_{l}-\varepsilon_{n}\right) t\right]\left[\psi_{n}^{*}(\mathbf{r}) \hat{\mathbf{p}} \psi_{l}(\mathbf{r})-\left(\hat{\mathbf{p}} \psi_{n}^{*}(\mathbf{r})\right) \psi_{l}(\mathbf{r})\right]\right\} \\
& -\frac{e^{2}}{m_{e} c}\left|\psi_{n}\right|^{2} \mathbf{A}(\mathbf{r}, t) .
\end{aligned}
$$

Then, applying the standard perturbation-theory technique [39] we obtain the equation describing the dynamics of the coefficients $\delta a_{l}(t)$ :

$$
\begin{aligned}
& i \sum \frac{\partial \delta a_{l}^{(n)}(t)}{\partial t} \psi_{l}(\mathbf{r}) \exp \left(-i \varepsilon_{l} t\right)=-\frac{e}{2 m_{e} c} \\
& \quad \times[\mathbf{A}(\mathbf{r}, t) \hat{\mathbf{p}}+\hat{\mathbf{p}} \mathbf{A}(\mathbf{r}, t)] \psi_{n}(\mathbf{r}) \exp \left(-i \varepsilon_{n} t\right),
\end{aligned}
$$

which is obtained by substituting Eq. (4) into the Schrödinger equation and its subsequent linearization with respect to the electromagnetic field strength. The Fourier transform of Eq. (7) gives

$$
\begin{aligned}
\delta a_{l}^{(n)}(\omega) & =\frac{e}{2 m_{e} \omega c}\langle l| \mathbf{A}\left(\mathbf{r}, \omega+\varepsilon_{l}-\varepsilon_{n}\right) \hat{\mathbf{p}} \\
& +\hat{\mathbf{p}} \mathbf{A}\left(\mathbf{r}, \omega+\varepsilon_{l}-\varepsilon_{n}\right)|n\rangle .
\end{aligned}
$$

Here we use the standard bra- and ket- notation for the wavefunctions and matrix elements, $|l\rangle=$ $\psi_{l}(\mathbf{r})$. Only those terms are preserved in Eq. (8) which correspond to the resonant interaction between electrons and electromagnetic field. The contribution of the last term in (6) is therefore neglected in Eq. (8). Performing the Fourier transform of Eq. (6) along the axial coordinate and time, we come to the $k, \omega$-space interaction-induced current density correction:

$$
\begin{aligned}
& \delta \mathbf{j}_{n}\left(k, \mathbf{r}_{\perp}, \omega\right)=-\frac{e^{2}}{4 m_{e}^{2} c} \sum_{l \tau^{\prime} \tau} B_{n l}\left(k, \mathbf{r}_{\perp}, \omega\right) \\
& \quad \times\left\{-\frac{b_{l \tau^{\prime}}^{*} b_{n \tau}\left[u_{l \tau^{\prime}}^{*}\left(\hat{\mathbf{p}}_{n}+\tau\right)+\left(\hat{\mathbf{p}}_{n}+\tau\right) u_{l \tau^{\prime}}^{*}\right] u_{n \tau}}{\omega+\varepsilon_{l}\left(p_{n}-k\right)-\varepsilon_{n}\left(p_{n}\right)}\right. \\
& \left.\quad+\frac{b_{n \tau}^{*} b_{l \tau^{\prime}}\left[u_{n \tau}^{*}\left(\hat{\mathbf{p}}_{n}+\tau\right)+\left(\hat{\mathbf{p}}_{n}+\tau\right) u_{n \tau}^{*}\right] u_{l \tau^{\prime}}}{\omega+\varepsilon_{n}\left(p_{n}\right)-\varepsilon_{l}\left(p_{n}+k\right)}\right\} .
\end{aligned}
$$


For convenience, we have introduced the vector form for the lattice constant $\tau: \tau=\tau \mathbf{e}_{z}$, where $\mathbf{e}_{z}$ is the unit axial vector. The quasi-momentum operator entering the matrix elements is given by $\hat{\mathbf{p}}_{n}=\left\{\hat{\mathbf{p}}_{\perp}, p_{n}\right\}$, where the axial components $p_{n}$ are $C$-numbers and transverse components $\hat{\mathbf{p}}_{\perp}$ are operators. These operators act only on the right-adjacent functions. Deriving Eq. (9), we neglected the longitudinal component $k$ of the electromagnetic wave vector in the matrix elements since $\hbar k / p_{n} \ll 1$. Summation over the lattice constants $\tau$ and $\tau^{\prime}$ is not independent: for every $\tau$ in sum, the value of $\tau^{\prime}$ must be such that the values $p_{n}+\tau-\tau^{\prime}$ are in the $\mathrm{f}$ rst Brillouin zone. The coeff cients $B_{n l}\left(k, \mathbf{r}_{\perp}, \omega\right)$ are given by

$$
\begin{aligned}
& B_{n l}\left(k, \mathbf{r}_{\perp}, \omega\right)=\sum_{\tau^{\prime} \tau} b_{l \tau^{\prime}} b_{n \tau}^{*}\left\langle u_{n \tau}\right|\left(\hat{\mathbf{p}}_{n}+\tau\right) \mathbf{A}\left(k, \mathbf{r}_{\perp}, \omega\right) \\
& \quad+\mathbf{A}\left(k, \mathbf{r}_{\perp}, \omega\right)\left(\hat{\mathbf{p}}_{n}+\tau\right)\left|u_{l \tau^{\prime}}\right\rangle
\end{aligned}
$$

Then by substituting Eq. (9) into Eq. (2) we come to a self-consistent field equation necessary for the further analysis.

\subsection{Dispersion equation for electromagnetic wave coupled with electron beam}

The electromagnetic response properties of an isolated single-wall CNT were studied in Ref. 35 on the base of a tight-binding microscopic model of the CNT conductivity and the effective boundary conditions for an electromagnetic field imposed on the CNT surface. A detailed analysis of the eigenwave problem has revealed that there are strongly slowed down surface waves propagating in CNTS leading to the concept of nanotubes as surface-wave nanowaveguides. Considering the electron beam as a perturbation, we can use the dispersion equation for the surface waves and the propagation constants obtained in Ref. 35 as a zero-order approximation. Then, the self-consistent field of the electromagnetic wave coupled with an electron beam can be presented by the expansion

$$
\mathbf{A}\left(k, \mathbf{r}_{\perp}, \omega\right)=\sum_{m} \alpha_{m}(k, \omega) \mathbf{A}_{m}\left(\mathbf{r}_{\perp}\right)
$$

where the vector potentials $\mathbf{A}_{m}\left(\mathbf{r}_{\perp}\right)$ correspond to the electromagnetic field eigenfunctions evaluated in Ref. 35 and $\alpha_{m}(k, \omega)$ are the coefficients to be found. Substitution of Eqs. (10), (9) and (6) into (2) gives a system of equations for the electromagnetic field interacting with the electrons occupying the $n$-th state:

$$
\begin{gathered}
\sum_{m}\left(k^{2}-k_{m}^{2}\right) \alpha_{m}(k, \omega) \mathbf{A}_{m}\left(\mathbf{r}_{\perp}\right)=-\frac{4 \pi}{c} \frac{e^{2} n_{e}}{4 m_{e}^{2} c} \sum_{l \tau^{\prime} \tau} B_{n l}\left(k, \mathbf{r}_{\perp}, \omega\right) \\
\quad \times\left\{-\frac{b_{l \tau^{\prime}}^{*} b_{n \tau}\left[u_{l \tau^{\prime}}^{*}\left(\hat{\mathbf{p}}_{n}+\tau\right)+\left(\hat{\mathbf{p}}_{n}+\tau\right) u_{l \tau^{\prime}}^{*}\right] u_{n \tau}}{\omega+\varepsilon_{l}\left(p_{n}-k\right)-\varepsilon_{n}\left(p_{n}\right)}\right. \\
\left.\quad+\frac{b_{n \tau}^{*} b_{l \tau^{\prime}}\left[u_{n \tau}^{*}\left(\hat{\mathbf{p}}_{n}+\tau\right)+\left(\hat{\mathbf{p}}_{n}+\tau\right) u_{n \tau}^{*}\right] u_{l \tau^{\prime}}}{\omega+\varepsilon_{n}\left(p_{n}\right)-\varepsilon_{l}\left(p_{n}+k\right)}\right\} .
\end{gathered}
$$

Here $k_{m}$ are the wavenumbers corresponding to the physical system devoid of the electron beam. As one can see, in deriving Eq. (11) we have proceeded from the single-electron dynamics to the dynamics of the electron beam: $n_{e}$ is the electron density. Multiplying left- and right-hand parts of Eq. (11) by $\mathbf{A}_{m}^{*}\left(\mathbf{r}_{\perp}\right)$ and utilizing the wavefunctions' orthogonality, we come to the dispersion equation as follows: 


$$
\begin{aligned}
& k-k_{m}=-\frac{\omega_{L}^{2}}{8 k_{m} m_{e} c^{2}} \sum_{l}\left|B_{n l}^{(m)}\right|^{2} \\
& \times\left[\frac{1}{-\omega+\varepsilon_{n}\left(p_{n}\right)-\varepsilon_{l}\left(p_{n}-k\right)}+\frac{1}{\omega+\varepsilon_{n}\left(p_{n}\right)-\varepsilon_{l}\left(p_{n}+k\right)}\right] .
\end{aligned}
$$

The upper index in $B_{n l}^{(m)}$ relates the matrix element with the corresponding mode of the electromagnetic field $\mathbf{A}_{m}\left(\mathbf{r}_{\perp}\right) ; \omega_{L}=2 \sqrt{\pi e^{2} n_{e} / m_{e}}$ is the Langmuir frequency of the electron beam. The transcendent dispersion equation (12) predicts the existence of a variety of branches of wavenumber $k$. Among them, the number of branches to be accounted for is defined by specific physical parameters of the analyzed system. In the vicinity of the resonance, only terms corresponding to the resonant interaction, one or several (in the case of degeneracy), can be kept in the dispersion equation. If the difference between levels exceeds the linewidth, only the resonant term is of importance.

\subsection{Classical and quantum limits in synchronism conditions}

The two terms in the right-hand side of Eq. (12) dictate two synchronism conditions corresponding to the resonant interaction between the electron beam and electromagnetic wave:

$$
\pm \omega+\varepsilon_{n}\left(p_{n}\right)-\varepsilon_{l}\left(p_{n} \pm k\right)=0 .
$$

The plus and minus signs in Eq. (13) correspond to the absorption and the emission of a photon by an electron, respectively. Depending on the relation between electron and photon energies, different interaction regimes are realized. As we restrict ourselves to the case when the photon momentum is much less than the electron one, the electron energy $\varepsilon_{l}\left(p_{n} \pm k\right)$ can be presented by the truncated Taylor series as

$$
\varepsilon_{l}\left(p_{n} \pm k\right)=\varepsilon_{l}\left(p_{n}\right) \pm k \frac{\partial \varepsilon_{l}\left(p_{n}\right)}{\partial p_{n}} \equiv \varepsilon_{l}\left(p_{n}\right) \pm k v_{l},
$$

where $v_{l}$ is the electron group velocity. Then, the denominators in (12) can be represented by

$$
\begin{aligned}
\pm \omega & +\varepsilon_{n}\left(p_{n}\right)-\varepsilon_{l}\left(p_{n} \pm k\right) \\
& \approx \pm\left(\omega-k v_{l} \pm \Omega_{n l}\right)+\frac{1}{2} \frac{\partial^{2} \varepsilon_{l}}{\partial p_{n}^{2}} k^{2} .
\end{aligned}
$$

The first term in the right-hand side of this equation is analogous to the standard term $\omega-k u \pm \Omega$ in the synchronism condition [26]. The only difference is that the velocity of the free electrons is replaced by the group velocity of quasi-electrons, $v_{l}$, and the undulation frequency is replaced by the transition frequency, $\Omega_{n l}=\varepsilon_{n}\left(p_{n}\right)-\varepsilon_{l}\left(p_{n}\right)$, between the CNT energy bands. The last term in Eq. (15) originates from the quantum recoil of an electron during the emission (absorption) of a photon and induces a red (blue) shift in the transition frequency. This term is inversely proportional to the electron effective mass (second derivative of the energy). Let $l=s$ be an electron level corresponding to the resonant interaction. Then, within the approximation stated, the dispersion equation takes the following form:

$$
k-k_{m}=\frac{2 b_{n s}^{(m)}\left(\frac{k^{2}}{2} \frac{\partial^{2} \varepsilon_{s}}{\partial p_{n}^{2}}-\Omega_{n s}\right)}{\left(\omega-k v_{s}\right)^{2}-\left(\frac{k^{2}}{2} \frac{\partial^{2} \varepsilon_{s}}{\partial p_{n}^{2}}-\Omega_{n s}\right)^{2}},
$$

where

$$
b_{n s}^{(m)}=-\frac{\omega_{L}^{2}}{8 m_{e} k_{m}^{\prime} c^{2}}\left|B_{n s}^{(m)}\right|^{2}, \quad k_{m}^{\prime}=\operatorname{Re}\left(k_{m}\right) .
$$


In the case of intraband transitions $\Omega_{n s}=0$ and Eq. (16) takes the form of the dispersion equation for the instability which takes into account recoil [16].

Depending on the ratio between the radiation linewidth and the recoil-induced detuning, two different generation regimes are realized. In the low-gain limit [18] the spontaneous emission linewidth can be estimated as $\Delta \omega / \omega \sim c /(\omega L)$, where $L$ is the interaction length. If the linewidth exceeds the recoil energy, the recoil term in the denominator of Eq. (16) can be neglected and the classical interaction regime is realized. The dispersion equation in that case takes the traditional form of the second-order Čerenkov resonance:

$$
k-k_{m}=k^{2} \frac{\partial^{2} \varepsilon_{s}}{\partial p_{n}^{2}} \frac{b_{n s}^{(m)}}{\left(\omega-k v_{s}\right)^{2}} .
$$

The spatial increment of the instability $k^{\prime \prime}=\operatorname{Im}(k)$ can be estimated using the method of weakly coupled modes [40]. According to this method, the interaction between the electromagnetic wave and the electron beam is essential only in the vicinity of the point $\left(\omega_{0}, k_{0}=\omega_{0} / v_{s}\right)$ where the dispersion curves of the noninteracting modes, $\omega-k v_{s}=0$ and $k(\omega)=k_{m}(\omega)$, are crossed. Then $k_{m}$ is represented by the expansion

$$
k_{m}(\omega)=k_{0}+\left.\frac{\partial k_{m}(\omega)}{\partial \omega}\right|_{\omega=\omega_{0}}\left(\omega-\omega_{0}\right) .
$$

Substituting this expansion and $k=k_{0}+\Delta k$ into Eq. (17) results in a third-order algebraic equation with respect to $\Delta k$. From this equation, the instability spatial increment is estimated at the frequency $\omega=\omega_{0}$ as

$$
\left|\Delta k^{\prime \prime}\right|=\frac{\sqrt{3}}{2}\left|b_{n n}^{(m)} \frac{\partial^{2} \varepsilon_{n}}{\partial p_{n}^{2}} \frac{k^{2}}{v_{n}^{2}}\right|^{1 / 3},
$$

where $\Delta k^{\prime \prime}=\operatorname{Im}(\Delta k)$. Since $b_{n n} \sim n_{e}$, the increment is found to be the 3-rd root of the electron density. Such a dependence is typical for the Compton-type radiative instability [18].

In the opposite case, when the linewidth is less then the difference between the emission and the absorption frequencies, we fall into the regime of strong quantum recoil impact. In this case, only the term corresponding to the emission survives in the dispersion equation (12), which therefore is reduced to

$$
k-k_{m}=b_{n n}^{(m)} \frac{1}{\omega-v_{s} k-\frac{1}{2} \frac{\partial^{2} \varepsilon_{n}}{\partial p_{n}^{2}} k^{2}} .
$$

As a result, the instability increment is given by

$$
\left|\Delta k^{\prime \prime}\right|=\left|\frac{b_{n n}^{(m)}}{v_{n}}\right|^{1 / 2},
$$

i.e., turns out to be proportional to the square root of the electron density.

Next we present a detail discussion of the different generation regimes and give some numerical estimates of physical parameters corresponding to these regimes.

\subsection{Boundary conditions for a finite-length nanotube}

In Secs. 2.2 and 2.3, dispersion equations have been derived providing us with wavenumber eigenvalues in an infinite-length CNT guiding an electron beam. As a next step, edge conditions must be imposed upon the system to account for the finite length of the interaction zone. 
These conditions are stated as the requirement for the perturbations of the electron and current densities, generated by the electron beam - electromagnetic wave interaction, to be zero at the input of the working zone, i.e.

$$
\delta n_{e}(z=0)=\delta j_{n}(z=0)=0 .
$$

The condition that the tangential electric f eld component and the axial component of the magnetic field be continuous on the CNT surface yields an additional boundary condition. We write it in the simplif ed form [41] as

$$
E(z=0)=\alpha E(z=L)
$$

where $\alpha$ is the ref ection coefficient of electromagnetic field from the working zone boundaries.

The field distribution in a finite-length system consisting of several parts can be found by solving electrodynamical problem in each region separately and then joining the solutions by means of the boundary conditions. In the interaction region, the electromagnetic field is given by

$$
E(z) \sim \sum_{i=1}^{N} c_{i} \exp \left(i k^{(i)} z\right),
$$

where the summation is performed over all electromagnetic modes in the CNT; the wavenumbers $k^{(i)}$ are determined by the corresponding dispersion equations. Note that the reflection of the electromagnetic waves from the boundaries back into the working zone creates positive feedback in the system and thus allows the accumulation of electromagnetic energy and provides an oscillator regime.

\subsection{Starting current at a large quantum recoil}

In the quantum interaction regime, when the quantum recoil exceeds the linewidth, the instability is described by the quadratic dispersion equation (20) with solutions $k^{(1)}$ and $k^{(2)}$. Consequently, the electric feld and the perturbation of the current density in the working zone are given by

$$
\begin{aligned}
& E \sim c_{1} \exp \left(i k^{(1)} z\right)+c_{2} \exp \left(i k^{(2)} z\right) \\
& \delta j_{n} \sim \frac{c_{1}}{\delta_{1}} \exp \left(i k^{(1)} z\right)+\frac{c_{2}}{\delta_{2}} \exp \left(i k^{(2)} z\right) .
\end{aligned}
$$

The coefficients

$$
\delta_{1,2}=1-\frac{v_{n}}{\omega} k^{(1,2)}+\frac{1}{2 \omega} \frac{\partial^{2} \varepsilon_{n}}{\partial p_{n}^{2}} k^{(1,2) 2}
$$

introduce deviations of the wavenumbers $k^{(1)}$ and $k^{(2)}$ from the synchronism, and the coefficients $c_{i}$ are determined from the boundary conditions as was discussed in Sect. 2.4. Using the boundary conditions (22) and (23), we arrive at the linear system for $c_{i}$ as follows:

$$
\begin{aligned}
& c_{1}+c_{2}=\alpha\left[c_{1} \exp \left(i k^{(1)} L\right)+c_{2} \exp \left(i k^{(2)} L\right)\right], \\
& \frac{c_{1}}{\delta_{1}}+\frac{c_{2}}{\delta_{2}}=0 .
\end{aligned}
$$

The nontrivial solution of this system is determined by the equation

$$
\delta_{1}\left[1-\alpha \exp \left(i k^{(1)} L\right)\right]-\delta_{2}\left[1-\alpha \exp \left(i k^{(2)} L\right)\right]=0 .
$$


The current density satisfying Eq. (29) is the threshold current density of the generation. To evaluate this quantity, the characteristic equation (29) must be solved together with Eq. (20). Substituting the roots

$$
k^{(1,2)}=k_{m, \mathrm{ch}}+\frac{b_{n n}^{(m)}}{v_{n}\left(k_{\mathrm{ch}}-k_{m}^{\prime}\right)}
$$

of the dispersion equation (20), with $k_{\mathrm{ch}}$ extracted from the synchronism condition $\omega-k_{\mathrm{ch}} v_{n}+$ $\left(k_{\mathrm{ch}}^{2} / 2\right) \partial^{2} \varepsilon_{n} / \partial p_{n}^{2}=0$, into Eq. (29) and solving the resulting equation with respect to the current density, we obtain

$$
\frac{b_{n n}^{(m)}}{v_{n}} L^{2} \frac{\sin ^{2} x}{x^{2}}=1-|\alpha|+L k_{m}^{\prime \prime}
$$

where

$$
x=\left(\omega-k_{m}^{\prime} v_{n}+\frac{k_{m}^{\prime 2}}{2} \frac{\partial^{2} \varepsilon_{n}}{\partial p_{n}^{2}}\right) \frac{L}{2 c}
$$

is the dimensionless off-synchronism parameter.

Physically, Eq. (31) establishes the energy balance in the working zone. Its left-hand side determines the radiation production, which is proportional to the electron density $n_{e}$ and to the squared interaction length. The factor $\sin ^{2} x / x^{2}$ determines the so called gain curve - the gain dependence on the off-synchronism parameter $x$. In the case considered the gain curve is symmetrical with respect to $x=0$ and is maximal at zero deviation $x$. Further we compare this result with the classical case of small recoil and demonstrate a significant difference in the behavior of gain curves. The term $1-|\alpha|$ in the right-hand side of Eq. (31) corresponds to the radiation leakage through the boundaries of the interaction zone while the last term specifies the radiation absorption by the nanotube.

The energy balance equation (31) allows the evaluation of the threshold current density. If the current density in CNT exceeds the threshold value, the generation process occurs. The characteristic time of the instability development is inversely proportional to the absolute instability increment $\omega^{\prime \prime}=\operatorname{Im}(\omega)$, which is derived by solving the generation equation (29) with respect to $\omega(k)$. In the low-gain regime [18], which implies the conditions $\left|\Delta k^{\prime \prime}\right| L \ll 1$ and $1-\alpha \ll 1$, the increment is given by:

$$
\omega_{m}^{\prime \prime}=\left[\frac{\partial k_{m}}{\partial \omega}\right]^{-1}\left(\frac{b_{n n}^{(m)}}{v_{n}} L \frac{\sin ^{2} x}{x^{2}}-\frac{1-|\alpha|}{L}-k_{m}^{\prime \prime}\right) .
$$

In the linear stage of the radiative instability development, the electromagnetic field grows with time as $\exp \left(\omega_{m}^{\prime \prime} t\right)$.

\subsection{Starting current in the classical regime of interaction}

In the case when quantum recoil can be neglected, the dispersion equation (17) has three roots

$$
\begin{gathered}
k^{(1)}=k_{m}-b_{n n}^{(m)} \frac{\partial^{2} \varepsilon_{n}}{\partial p_{n}^{2}} \frac{k_{m}^{\prime 2}}{\left(\omega-v_{n} k_{m}^{\prime}\right)^{2}} \\
k^{(2,3)}=k_{\mathrm{ch}} \pm \frac{i}{v_{n}} \sqrt{b_{n n}^{(m)} \frac{\partial^{2} \varepsilon_{n}}{\partial p_{n}^{2}} \frac{k_{m}^{\prime 2}}{k_{\mathrm{ch}}-k_{m}^{\prime}}} .
\end{gathered}
$$

and, consequently, the electromagnetic field in the interaction region is given by Eq. (24) with $N=3$. Correspondingly, perturbations of the electron and the current densities in the beam are 
written as

$$
\delta j_{n} \sim \sum_{i=1}^{3} \frac{c_{i}}{\nu_{i}^{2}}, \quad \delta j_{n}-v_{n} \delta n_{e} \sim \sum_{i=1}^{3} \frac{c_{i}}{\nu_{i}}
$$

where deviations $\nu_{i}$ are given by Eq. (27) with the last term omitted, i.e., $\nu_{i}=1-k^{(i)} v_{n} / \omega$. Then, by analogy with the previous section, we obtain the linear system

$$
\begin{aligned}
c_{1}+ & c_{2}+c_{3}=\alpha\left[c_{1} \exp \left(i k^{(1)} L\right)\right. \\
& \left.+c_{2} \exp \left(i k^{(2)} L\right)+c_{3} \exp \left(i k^{(3)} L\right)\right], \\
\frac{c_{1}}{\nu_{1}}+ & \frac{c_{2}}{\nu_{2}}+\frac{c_{3}}{\nu_{3}}=0 \\
\frac{c_{1}}{\nu_{1}^{2}}+ & \frac{c_{2}}{\nu_{2}^{2}}+\frac{c_{3}}{\nu_{3}^{2}}=0
\end{aligned}
$$

and corresponding generation equation

$$
\begin{aligned}
& \nu_{1}^{2}\left(\nu_{2}-\nu_{3}\right)\left[1-\alpha \exp \left(i k^{(1)} L\right)\right] \\
& -\nu_{2}^{2}\left(\nu_{1}-\nu_{3}\right)\left[1-\alpha \exp \left(i k^{(2)} L\right)\right] \\
& +\nu_{3}^{2}\left(\nu_{1}-\nu_{2}\right)\left[1-\alpha \exp \left(i k^{(3)} L\right)\right]=0 .
\end{aligned}
$$

We solve this equation in the low gain limit, which is determined by the condition $k_{z}^{\prime \prime} L \leq 1$.

Then, solutions of Eq. (37) (the threshold current and the temporal instability increment) are given by

$$
\begin{aligned}
\frac{b_{n n}^{(m)}}{v_{n}^{2}} & \frac{\partial^{2} \varepsilon_{n}}{\partial p_{n}^{2}} k L^{3} \frac{x \cos x-\sin x}{x^{3}}=1-|\alpha|+L k_{m}^{\prime \prime}, \\
\omega_{m}^{\prime \prime} & =\left[\frac{\partial k_{m}}{\partial \omega}\right]^{-1}\left[\frac{b_{n n}^{(m)}}{v_{n}^{2}} \frac{\partial^{2} \varepsilon_{n}}{\partial p_{n}^{2}} L^{2} \frac{x \cos x-\sin x}{x^{3}}\right. \\
& \left.-\frac{1-|\alpha|}{L}+k_{m}^{\prime \prime}\right]
\end{aligned}
$$

with the parameter $x$ defined by Eq. (32). As follows from the balance equations (31) and (38), in the quantum interaction regime the radiation production per unit length is characterized by the linear dependence on $L$, while this dependence becomes quadratic in the classical regime. The gain curves display distinctive behavior in these two cases. In contrast to the quantum interaction regime, the classical limit yields a gain curve, which is asymmetric [18] due to the interference of absorption and emission processes separated by a frequency gap narrower than the linewidth. As a result, the sign of the absolute instability increment depends on the sign of the synchronism detuning. At positive detuning the system is closer to the absorption frequency while negative detuning moves the system to the emission frequency. The simplest way to realize a nanoFEL in a carbon nanotube is to inject a high energy external electron beam. Since the velocity of free electrons is $v(\mathrm{~cm} / \mathrm{s})=5.7 \times 10^{7} \sqrt{\varphi(\mathrm{eV})}$, in order to accelerate electrons up to the velocities required for the synchronism regime (with 50-100 times wave slowing down predicted in Ref. 35, it is necessary to apply a voltage of $\varphi \sim 7 \mathrm{eV}$. If the CNT diameter is such that its product with the electron transversal momentum is $p_{\perp} D \sim 10-100$, the electron 
motion can be treated as classical. In that case, the term in the right-hand side of the dispersion equation (17) can be modified in the following way

$$
b_{n n}^{(m)} \frac{\partial^{2} \varepsilon_{n}}{\partial p_{n}^{2}} \frac{k^{2}}{\left(\omega-v_{n} k\right)^{2}} \sim \omega_{L}^{2} \frac{(\mathbf{v e})^{2}}{2 k_{m}^{\prime} c^{2}} \frac{k^{2}}{(\omega-v k)^{2}} .
$$

where $\mathbf{v}$ is the classical electron velocity and $\mathbf{e}$ is polarization vector for the electromagnetic mode considered. This simplification, after substitution of Eq. (40) into Eqs. (38) and (39), allows us to estimate the threshold current required to start the generation process and the instability increment, respectively. The dependences of these quantities on the CNT length are depicted in Figs. 1 and 2. Calculations have been done for $1 \mu \mathrm{m}$ radiation wavelength and for the reflection coefficient from the working zone boundaries $\alpha=0.99$. Generation in the terahertz range would require higher current density. The gain for CNTs is extremely large

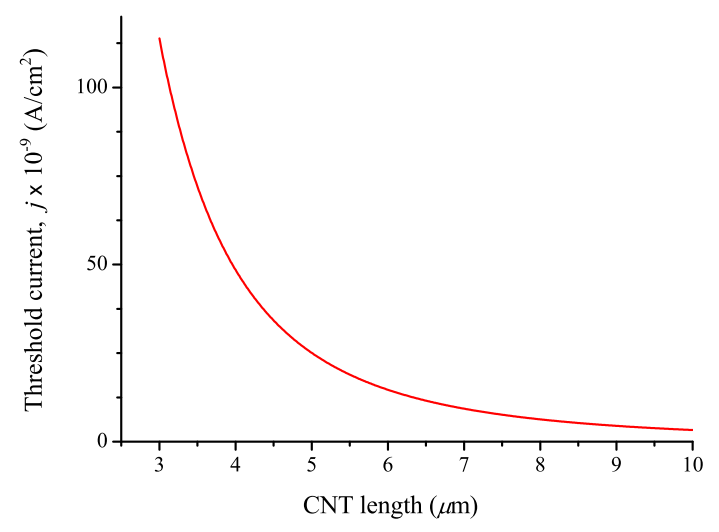

Fig. 1. The dependence of threshold current density on nanotube length.

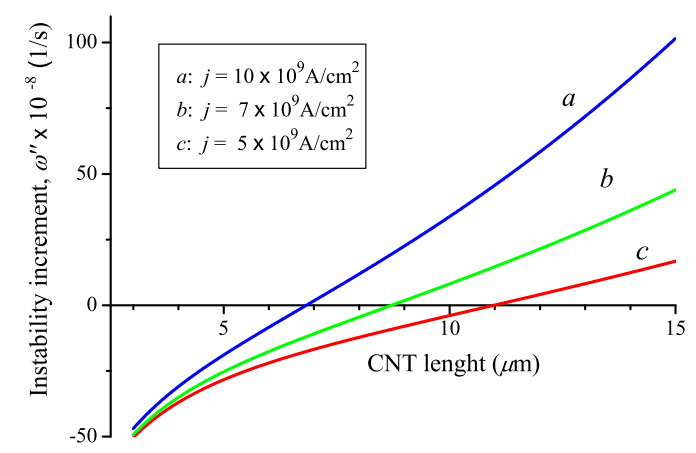

Fig. 2. Instability increment vs nanotube length at different electron current densities.

compared to macroscopic electronic devices. For the chosen parameters, the generation development starts when the CNT length is about $6 \mu \mathrm{m}$ or larger, which is technologically attainable length. Therefore, our calculations demonstrate that the development of CNT-based nanoFEL 
is already possible for the current stage of nanotechnology. The characteristic time of the instability evolution is inversely proportional to the instability increment and for a $10 \mu \mathrm{m}$ nanotube is a fraction of nanosecond.

\section{RETARDATION OF ELECTROMAGNETIC WAVES IN MULTI-WALL CARBON NANOTUBES}

The typical group velocity of $\pi$-electrons in SWNT is considerably smaller $(c / v>100)$ than the phase velocity of the retarded electromagnetic wave; these velocities must coincide to provide effective Cerenkov generation. Therefore, methods and systems providing additional slowing of the electromagnetic wave is needed. In this section we consider the wave retardation in multiwall CNTs (MWNTs) and graphene. We show that the interaction between nanotube walls leads to considerable modif cation of the electromagnetic wave dispersion and reduction of the phase velocity.

The D'Alembert equation for the electric potential has the form:

$$
\left(\frac{\partial^{2}}{c^{2} \partial t^{2}}-\Delta\right) \Phi(\mathbf{r}, t)=4 \pi \rho(\mathbf{r}, t)
$$

where $\Phi(\mathbf{r}, t)$ is the scalar potential and $\rho(\mathbf{r}, t)$ is the electric charge density. For the strongly slowed-down wave, the inequality $\omega^{2} / c^{2} \ll k^{2}$ holds true. Therefore the time derivatives are much less than the spatial ones: $\frac{\partial^{2} \Phi}{c^{2} \partial t^{2}} \ll \Delta \Phi$. In this case, the D'Alembert equation is reduced to the Poisson equation

$$
\Delta \Phi(\mathbf{r}, t)=-4 \pi \rho(\mathbf{r}, t),
$$

which is used in the further analysis. Equation (42) is supplemented by the equation describing the electric charge density:

$$
\rho(\mathbf{r}, t)=\sum_{i} \rho_{i}\left(\mathbf{r}_{i \perp, t}\right) \delta\left(r-R_{i}\right)=e \sum_{i}\left\langle 0\left|\psi_{i}^{+}\left(\mathbf{r}_{\perp}, t\right) \psi_{i}\left(\mathbf{r}_{\perp}, t\right)\right| 0\right\rangle_{i} \delta\left(r-R_{i}\right) .
$$

Here, $\psi_{i}\left(\mathbf{r}_{\perp}, t\right)$ is the electron wave function on $\mathrm{i}$-th nanotube wall in the secondary quantization representation. This function is expressed in terms of the electron annihilation operators, $\rho_{i}\left(\mathbf{r}_{\perp}, \mathbf{t}\right)$ is two-dimensional charge density of $\mathbf{i}$-th $\mathrm{CNT}$ wall, $\mathbf{r}_{\perp}$ is two-dimensional vector on CNT walls $\left(\mathbf{r}=\left(r, \mathbf{r}_{\perp}\right)\right), r$ is the radial component of $\mathbf{r}, R_{i}$ is the wall radius.

$$
\psi_{i}\left(\mathbf{r}_{\perp}, t\right)=\sum \psi_{k s}^{(i)}\left(\mathbf{r}_{\perp}\right) b_{k s}^{(i)}(t)
$$

where $\psi_{k s}^{(i)}$ is the electron Bloch wave function in the CNT, $b_{k s}^{(i)}$ is the annihilation operator for the corresponding state of i-th wall, $k, s$ are electron quasi-momentum and index of energy band, $|0\rangle_{i}$ is the ground state of the electron subsystem of $\mathrm{i}$-th wall.

The dynamics of the charge density is completely described by the dynamics of the creation and annihilation operators, which is governed by the Hamiltonian as follows:

$$
H=\sum_{i k s} E_{k s}^{(i)} b_{k s}^{(i)+} b_{k s}^{(i)}+\left.e \sum_{i} \int \Phi(\mathbf{r}, t)\right|_{r=R_{i}} \psi_{i}^{+}\left(\mathbf{r}_{\perp}, t\right) \psi_{i}\left(\mathbf{r}_{\perp}, t\right) d \mathbf{r}_{\perp} .
$$

The following equations describing the dynamics of the operators can be produced by using Eq. (45):

$$
\begin{aligned}
& \dot{b}_{k s}^{(j)}(t)=-i E_{k s}^{(j)} b_{k s}^{(j)}(t)-i e \sum_{k_{1} s_{1} j}\left\langle k s|\Phi(\mathbf{r}, t)|_{r=R_{j}} \mid k_{1} s_{1}\right\rangle_{j} b_{k_{1} s_{1}}^{(j)}, \\
& \dot{b}_{k s}^{(j)+}(t)=i E_{k s}^{(j)} b_{k s}^{(j)+}(t)+i e \sum_{k_{1} s_{1} j}\left\langle k_{1} s_{1}|\Phi(\mathbf{r}, t)|_{r=R_{j}} \mid k s\right\rangle_{j} b_{k_{1} s_{1}}^{(j)+} .
\end{aligned}
$$


The solution of Eq. (46) without electromagnetic wave accounting has the apparent, trivial form: $b_{k s}^{(j)}(t)=\mathrm{b}_{k s}^{(j)} \exp \left\{-i E_{k s}^{(j)} t\right\}$. Let us make substitution $b_{k s}^{(j)}(t)=\mathrm{b}_{k s}^{(j)}(t) \exp \left\{-i E_{k s}^{(j)} t\right\}$. Then, Eq. (46) can be reduced to

$$
\begin{gathered}
\frac{d \mathrm{~b}_{k s}^{(j)}(t)}{d t}=-i e \sum_{k_{1} s_{1}}\left\langle k s|\Phi(\mathbf{r}, t)|_{r=R_{j}} \mid k_{1} s_{1}\right\rangle_{j} \mathrm{~b}_{k_{1} s_{1}} \exp \left\{i\left(E_{k s}^{(j)}-E_{k_{1} s_{1}}^{(j)}\right) t\right\}, \\
\frac{d \mathrm{~b}_{k s}^{(j)+}(t)}{d t}=i e \sum_{k_{1} s_{1}}\left\langle k_{1} s_{1}|\Phi(\mathbf{r}, t)|_{r=R_{j}} \mid k s\right\rangle_{j} \mathrm{~b}_{k_{1} s_{1}}^{(j)+} \exp \left\{-i\left(E_{k s}^{(j)}-E_{k_{1} s_{1}}^{(j)}\right) t\right\} .
\end{gathered}
$$

Considering electromagnetic field in Eq. (47) as a perturbation and expanding operators $\mathrm{b}_{k s}^{(i)}=\mathrm{b}_{k s}^{(i 0)}+\delta \mathrm{b}_{k s}^{(i)}$, we easy to produce, in linear approximation, the following expressions for the temporal Fourier component of annihilation and creation operators:

$$
\begin{aligned}
& \delta \mathrm{b}_{k s}^{(i)}(\omega)=\frac{e}{\omega} \sum_{k_{1} s_{1}} \mathrm{~b}_{k_{1} s_{1}}^{(i 0)}\left\langle k s\left|\Phi\left(\mathbf{r}, \omega+E_{k s}^{(i)}-E_{k_{1} s_{1}}^{(i)}\right)\right|_{r=R_{i}} \mid k_{1} s_{1}\right\rangle_{i}, \\
& \delta \mathbf{b}_{k s}^{(i)+}(\omega)=-\frac{e}{\omega} \sum_{k_{1} s_{1}} \mathrm{~b}_{k_{1} s_{1}}^{(i 0)+}\left\langle k_{1} s_{1}\left|\Phi\left(\mathbf{r}, \omega-E_{k s}^{(i)}+E_{k_{1} s_{1}}^{(i)}\right)\right|_{r=R_{i}} \mid k s\right\rangle_{i} .
\end{aligned}
$$

Substituting Eq. (48) in the expression (43) for electric charge density yields, in linear approximation,

$$
\begin{aligned}
& \rho_{\perp}^{(i)}\left(\mathbf{r}_{\perp}, \omega\right)=e \sum_{1,2} \psi_{k_{2} s_{2}}^{(i) *}\left(\mathbf{r}_{\perp}\right) \psi_{k_{1} s_{1}}^{(i)}\left(\mathbf{r}_{\perp}\right) \frac{\left\langle k_{1} s_{1}|\Phi(\mathbf{r}, \omega)|_{r=R_{i}} \mid k_{2} s_{2}\right\rangle_{i}}{\omega+E_{k_{2} s_{2}}^{(i)}-E_{k_{1} s_{1}}^{(i)}} \\
& \quad \times\left\langle 0\left|\mathrm{~b}_{k_{2} s_{2}}^{(i 0)+} \mathrm{b}_{k_{2} s_{2}}^{(i 0)}-\mathrm{b}_{k_{1} s_{1}}^{(i 0)+} \mathrm{b}_{k_{1} s_{1}}^{(i 0)}\right| 0\right\rangle_{i} .
\end{aligned}
$$

As a result, the Poisson equation (42) can be written in the closed form relative to the electric potential as:

$$
\begin{aligned}
& \Delta \Phi(\mathbf{r}, t)=-4 \pi e^{2} \sum_{i} \sum_{1,2} \psi_{k_{2} s_{2}}^{(i) *}\left(\mathbf{r}_{\perp}\right) \psi_{k_{1} s_{1}}^{(i)}\left(\mathbf{r}_{\perp}\right) \frac{\left\langle k_{1} s_{1}|\Phi(\mathbf{r}, \omega)|_{r=R_{i}} \mid k_{2} s_{2}\right\rangle_{i}}{\omega+E_{k_{2} s_{2}}^{(i)}-E_{k_{1} s_{1}}^{(i)}} \\
& \times\left\langle 0\left|\mathrm{~b}_{k_{2} s_{2}}^{(i 0)+} \mathrm{b}_{k_{2} s_{2}}^{(i 0)}-\mathrm{b}_{k_{1} s_{1}}^{(i 0)+} \mathrm{b}_{k_{1} s_{1}}^{(i 0)}\right| 0\right\rangle_{i} \delta\left(r-R_{i}\right)
\end{aligned}
$$

In Eq. (49), $R_{i}$ is the radius of the $i^{\text {th }}$ wall of MWNT. By neglecting the diffraction and performing the Fourier transform of equation (49) by the longitudinal coordinate $z$ and by the angle $\varphi$, we reduce Eq. (49) to the following form:

$$
\begin{aligned}
& \frac{1}{r} \frac{d}{d r}\left(r \frac{d \Phi(r, k, \omega, m)}{d r}\right)-\left(k^{2}+\frac{m^{2}}{r^{2}}\right) \Phi(r, k, \omega, m) \\
& =-4 \pi e^{2} \sum_{i} \sum_{1,2} \frac{\left|a_{k_{2} s_{2} \tau_{2} m_{2}}^{(i)}\right|^{2}\left|a_{k_{1} s_{1} \tau_{1} m_{1}}^{(i)}\right|^{2}}{\omega+E_{k_{2} s_{2}}^{(i)}-E_{k_{1} s_{1}}^{(i)}} \Phi(r, k, \omega, m) \delta\left(r-R_{i}\right) \\
& \times\left\langle 0\left|\mathrm{~b}_{k_{2} s_{2}}^{(i 0)+} \mathrm{b}_{k_{2} s_{2}}^{(i 0)}-\mathrm{b}_{k_{1} s_{1}}^{(i 0)+} \mathrm{b}_{k_{1} s_{1}}^{(i 0)}\right| 0\right\rangle_{i} .
\end{aligned}
$$

In Eq. (50), $\left\{a_{k s \tau m}^{(i)}\right\}$ are the coefficients in the expansion of corresponding electron Bloch functions. The wave numbers and azimuthal numbers of the initial and final states satisfy the equations

$$
\begin{gathered}
k_{2}=k_{1}+\tau_{1}-\tau_{2}-k, \\
s_{2}=s_{1}+m_{1}-m_{2}-m .
\end{gathered}
$$


If an additional electron beam propagates in the nanotube then instead of ground state $|0\rangle_{i}$, the state $\left|0, k_{b}\right\rangle_{i}$ should be used in Eq. (50) and the matrix element $\left\langle 0\left|\mathrm{~b}_{k_{2} s_{2}}^{(i 0)+} \mathrm{b}_{k_{2} s_{2}}^{(i 0)}-\mathrm{b}_{k_{1} s_{1}}^{(i 0)+} \mathrm{b}_{k_{1} s_{1}}^{(i 0)}\right| 0\right\rangle_{i}$ should be replaced by $\left\langle 0, k_{b}\left|\mathrm{~b}_{k_{2} s_{2}}^{(i 0)+} \mathrm{b}_{k_{2} s_{2}}^{(i 0)}-\mathrm{b}_{k_{1} s_{1}}^{i(0)+} \mathrm{b}_{k_{1} s_{1}}^{(i 0)}\right| 0, k_{b}\right\rangle_{i}$. Here $k_{b}$ are the quantum numbers describing the electron beam.

\subsection{Boundary conditions and the dispersion equation for an electromagnetic wave in a MWNT}

At $r \neq R_{i}$ the solution of Eq. (50) for the electric potential $\Phi(r, k, \omega, m)$ has the following form

$$
\begin{array}{ll}
A_{1} I_{m}(k r) & r<R_{1} \\
A_{i} I_{m}(k r)+B_{i} K_{m}(k r) & R_{i}<r<R_{i+1} \\
B_{N+1} K_{m}(k r) & r>R_{N} .
\end{array}
$$

Here $R_{i}$ is the radius of the $i^{t h}$ MWNT wall, $R_{1}$ is radius of the inner wall and $R_{N}$ is the radius of the outer wall. $I_{m}(k r)$ and $K_{m}(k r)$ are the modified Bessel function. Such form of solution satisfies finiteness of electromagnetic field in the whole coordinate space. $A_{i}$ and $B_{i}$ are the coefficients, which should be defined by using boundary conditions on the MWNT walls. These conditions can be written as:

$$
\begin{aligned}
& \Phi\left(R_{i}+0, k, \omega, m\right)=\Phi\left(R_{i}-0, k, \omega, m\right) \\
& \frac{d \Phi\left(R_{i}+0, k, \omega, m\right)}{d r}-\frac{d \Phi\left(R_{i}-0, k, \omega, m\right)}{d r}=-4 \pi e^{2} \sum_{1,2} \\
& \frac{\left|a_{k_{2} s_{2} \tau_{2} m_{2}}^{(i)}\right|^{2}\left|a_{k_{1} s_{1} \tau_{1} m_{1}}^{(i)}\right|^{2} \Phi\left(R_{i}, k, \omega, m\right)}{\omega+E_{k_{2} s_{2}}^{(i)}-E_{k_{1} s_{1}}^{(i)}} \times\left\langle 0\left|\mathrm{~b}_{k_{2} s_{2}}^{(i 0)+} \mathrm{b}_{k_{2} s_{2}}^{(i 0)}-\mathrm{b}_{k_{1} s_{1}}^{(i 0)+} \mathrm{b}_{k_{1} s_{1}}^{(i 0)}\right| 0\right\rangle_{i} .
\end{aligned}
$$

The first condition in Eq. (53) corresponds to continuity of the electric potential. The second boundary condition reflects discontinuity of the potential derivative due to surface charge on the walls. This condition is derived by the integration of (50) in the vicinity of the wall. Substituting Eq. (52) in Eq. (53) gives the following system for the coefficients $A_{i}$ and $B_{i}$ :

$$
\left\{\begin{array}{l}
A_{i+1} I_{m}\left(q R_{i}\right)+B_{i+1} K_{m}\left(q R_{i}\right)=A_{i} I_{m}\left(q R_{i}\right)+B_{i} K_{m}\left(q R_{i}\right) \\
A_{i+1} \frac{d I_{m}\left(q R_{i}\right)}{d r}+B_{i+1} \frac{d K_{m}\left(q R_{i}\right)}{d r}-A_{i} \frac{d I_{m}\left(q R_{i}\right)}{d r}-B_{i} \frac{d K_{m}\left(q R_{i}\right)}{d r}= \\
-4 \pi e^{2} \sum_{1,2} \frac{\left|a_{k_{2} s_{2} \tau_{2} m_{2}}^{(i)}\right|^{2}\left|a_{k_{1} s_{1} \tau_{1} m_{1}}^{(i)}\right|^{2}}{\omega+E_{k_{2} s_{2}}^{(i)}-E_{k_{1} s_{1}}^{(i)}}\left\langle 0\left|\mathrm{~b}_{k_{2} s_{2}}^{(i 0)+} \mathrm{b}_{k_{2} s_{2}}^{(i 0)}-\mathrm{b}_{k_{1} s_{1}}^{(i 0)+} \mathrm{b}_{k_{1} s_{1}}^{(i 0)}\right| 0\right\rangle_{i} \\
\quad \times\left(A_{i} I_{m}\left(q R_{i}\right)+B_{i} K_{m}\left(q R_{i}\right)\right) .
\end{array}\right.
$$

\subsection{Dispersion equations for waves in MWNTs}

The dependence of the coefficients in the $(i+1)^{t h}$ wall on the coefficients in the $i^{t h}$ wall can be derived from Eq. (54) and it has the form:

$$
\begin{aligned}
& A_{i+1}=A_{i}-f_{i} I_{m}\left(k R_{i}\right) K_{m}\left(k R_{i}\right) A_{i}-f_{i} K_{m}\left(k R_{i}\right) K_{m}\left(k R_{i}\right) B_{i} \\
& B_{i+1}=B_{i}+f_{i} I_{m}\left(k R_{i}\right) I_{m}\left(k R_{i}\right) A_{i}-f_{i} K_{m}\left(q R_{i}\right) I_{m}\left(q R_{i}\right) B_{i} .
\end{aligned}
$$


Here

$$
f_{i}=-4 \pi e^{2} \sum_{1,2} \frac{\left|a_{k_{2} s_{2} \tau_{2} m_{2}}^{(i)}\right|^{2}\left|a_{k_{1} s_{1} \tau_{1} m_{1}}^{(i)}\right|^{2}}{\omega+E_{k_{2} s_{2}}^{(i)}-E_{k_{1} s_{1}}^{(i)}}\left\langle 0\left|\mathrm{~b}_{k_{2} s_{2}}^{(i 0)+} \mathrm{b}_{k_{2} s_{2}}^{(i 0)}-\mathrm{b}_{k_{1} s_{1}}^{(i 0)+} \mathrm{b}_{k_{1} s_{1}}^{(i 0)}\right| 0\right\rangle .
$$

The linear system (55) must be supplemented by the conditions $A_{1}=0, B_{N+1}=0$, where $N$ is the number of nanotube walls. These conditions are required to guarantee finiteness of the electromagnetic field across the whole of space. The linear system (55) has non-zero solutions if its determinant is equal to zero. Equating the determinant to zero gives the dispersion equation for electromagnetic waves in nanotubes. For example, in the case of double-wall nanotube

$$
\begin{aligned}
& {\left[1+f_{1} I_{m}\left(k R_{1}\right) K_{m}\left(k R_{1}\right)\right]\left[1+f_{2} I_{m}\left(k R_{2}\right) K_{m}\left(k R_{2}\right)\right]} \\
& -f_{1} f_{2} K_{m}\left(k R_{2}\right) K_{m}\left(k R_{2}\right) I_{m}\left(k R_{1}\right) I_{m}\left(k R_{1}\right)=0 .
\end{aligned}
$$

In the limiting case when the electron system is the classical motionless plasma, the dispersion equation (56) has the following form

$$
\begin{aligned}
& {\left[1-\frac{\omega_{L 1}^{2}\left(m^{2}+k^{2} R_{1}^{2}\right) I_{m}\left(k R_{1}\right) K_{m}\left(k R_{1}\right)}{\omega^{2} R_{1}}\right]\left[1-\frac{\omega_{L 2}^{2}\left(m^{2}+k^{2} R_{2}^{2}\right) I_{m}\left(k R_{2}\right) K_{m}\left(k R_{2}\right)}{\omega^{2} R_{2}}\right]} \\
& -\frac{\omega_{L 2}^{2} \omega_{L 1}^{2}\left(m^{2}+k^{2} R_{2}^{2}\right)\left(m^{2}+k^{2} R_{1}^{2}\right)}{\omega^{4} R_{1} R_{2}} K_{m}\left(k R_{2}\right) K_{m}\left(k R_{2}\right) I_{m}\left(k R_{1}\right) I_{m}\left(k R_{1}\right)=0 .
\end{aligned}
$$

\subsection{Tight-binding method for deriving the wave dispersion equation}

The electron wave function in a MWNT was considered as being concentrated in the nanotube layers. But electrons can tunnel from one layer to another. Let us derive the dispersion equation taking account such tunneling for multi-layer graphene. Now the electron wave function is considered as three dimensional and isn't localized on layers. Then Eq. (49) is replaced by

$$
\begin{aligned}
& \Delta \Phi(\mathbf{r}, t)=-4 \pi e^{2} \sum_{i} \sum_{1,2} \psi_{k_{2} s_{2}}^{*}(\mathbf{r}) \psi_{k_{1} s_{1}}(\mathbf{r}) \frac{\left\langle k_{1} s_{1}|\Phi(\mathbf{r}, \omega)| k_{2} s_{2}\right\rangle}{\omega+E_{k_{2} s_{2}}-E_{k_{1} s_{1}}} \\
& \times\left\langle 0\left|\mathrm{~b}_{k_{2} s_{2}}^{(0)+} \mathrm{b}_{k_{2} s_{2}}^{(0)}-\mathrm{b}_{k_{1} s_{1}}^{(0)+} \mathrm{b}_{k_{1} s_{1}}^{(0)}\right| 0\right\rangle .
\end{aligned}
$$

The electron wave function in a monolayer or in a few layers of graphene in the tight-binding approximation can be written as

$$
\psi_{k s}=\sum c_{A_{i}} \psi_{k}^{A_{i}}(\mathbf{r})+\sum c_{B_{i}} \psi_{k}^{B_{i}}(\mathbf{r})
$$

Here $\psi_{k}^{A_{i}}(\mathbf{r}), \psi_{k}^{B_{i}}(\mathbf{r})$ are two tight-binding Bloch functions per layer. There are two types (A and $\mathrm{B}$ ) of carbon atoms in each graphene plane $i$, hence,

$$
\begin{aligned}
& \psi_{k}^{A_{i}}(\mathbf{r})=\frac{1}{\sqrt{N}} \sum_{A_{i}} \phi_{A}\left(\mathbf{r}-\mathbf{r}_{A_{i}}\right) \exp \left\{i \mathbf{k r}_{A_{i}}\right\}, \\
& \psi_{k}^{B_{i}}(\mathbf{r})=\frac{1}{\sqrt{N}} \sum_{B_{i}} \phi_{B}\left(\mathbf{r}-\mathbf{r}_{B_{i}}\right) \exp \left\{i \mathbf{k r}_{B_{i}}\right\} .
\end{aligned}
$$

Here $\mathbf{r}_{A_{i}}$ and $\mathbf{r}_{B_{i}}$ are the positions of the $\mathrm{A}$ and $\mathrm{B}$ carbon atoms in layer $i, \phi_{A}$ and $\phi_{B}$ denote the atomic wave functions of $A$ and $B$ carbon atoms, $N$ is the number of unit cells.

There are two wave functions corresponding to each wave number $\mathbf{k}$ in single-layer graphene [37] (bonding and anti-bonding solutions for $\pi$ electrons and two graphene bands which correspond to these solutions). For bilayer graphene the same method leads to four solutions. Electrons with two wave functions are concentrated near one graphene layer, and with two 
other wave functions are concentrated near the different layer. The Fourier transform over the transverse coordinate of Eq. (58) with this set of wave functions gives

$$
\begin{gathered}
\left(\frac{d^{2}}{d z^{2}}-k^{2}\right) \Phi=-4 \pi e^{2} \sum_{1,2} \int d \mathbf{r}_{\perp} \exp \left\{-i \mathbf{k r}_{\perp}\right\} \psi_{k_{2} s_{2}}^{*} \psi_{k_{1} s_{1}} \times \\
\frac{\left\langle k_{1} s_{1}|\Phi(\mathbf{r}, \omega)| k_{2} s_{2}\right\rangle}{\omega+E_{k_{2} s_{1}}-E_{k_{1} s_{2}}}\left\langle 0\left|b_{k_{2} s_{2}}^{(0)+} b_{k_{2} s_{2}}^{(0)}-b_{k_{1} s_{1}}^{(0)+} b_{k_{1} s_{1}}^{(0)}\right| 0\right\rangle .
\end{gathered}
$$

Using relations for the matrix elements with tight-binding functions $\left\langle\psi_{k_{1}}^{A_{i}}(\mathbf{r})|\Phi(\mathbf{r}, \omega)| \psi_{k_{2}}^{A_{i}}(\mathbf{r})\right\rangle=$ $\int d z \Phi(z, k, \omega) F_{A}(z, \mathbf{k})$, where $F_{A}(z, \mathbf{k})=\int d \mathbf{r}_{\perp} \exp \left\{-i \mathbf{k r}_{\perp}\right\}\left|\phi_{A}(\mathbf{r})\right|^{2}$ is the form-factor and using similar relations for the $\left\langle\psi_{k_{1}}^{A_{i}}(\mathbf{r})|\Phi(\mathbf{r}, \omega)| \psi_{k_{2}}^{B_{j}}(\mathbf{r})\right\rangle$, we can transform (61) to the form

$$
\begin{gathered}
\left(\frac{d^{2}}{d z^{2}}-k^{2}\right) \Phi=\sum_{i} f_{A_{i}}(z) \int \rho_{A_{i}}(z) d z \Phi(z, k, \omega)+ \\
\sum_{i} f_{B_{i}}(z) \int \rho_{B_{i}}(z) d z \Phi(z, k, \omega)=\sum_{i} A_{i} f_{A_{i}}(z)+B_{i} f_{B_{i}}(z) .
\end{gathered}
$$

Here $A_{i}=\int \rho_{A_{i}}(z) d z \Phi(z, \mathbf{k}, \omega)$ and $B_{i}=\int \rho_{B_{i}}(z) d z \Phi(z, \mathbf{k}, \omega), \rho_{A_{i}}=\left|\phi_{A_{i}}(\mathbf{r})\right|^{2}, \rho_{B_{i}}=$ $\left|\phi_{B_{i}}(\mathbf{r})\right|^{2}$ are the electron densities. The solution of Eq. (62) which is confined to the graphene plane has the form

$$
\begin{gathered}
\Phi(z, \mathbf{k})=\exp \{i k z\} \int_{+\infty}^{z} d z \exp \{-i k z\} \sum_{i} \frac{A_{i} f_{A_{i}}(z)+B_{i} f_{B_{i}}(z)}{2 k}- \\
\exp \{-i k z\} \int_{-\infty}^{z} d z \exp \{i k z\} \frac{A_{i} f_{A_{i}}(z)+B_{i} f_{B_{i}}(z)}{2 k} .
\end{gathered}
$$

Substituting Eq. (63) in $A_{i}=\int \rho_{A_{i}}(z) d z \Phi(z, \mathbf{k}, \omega)$ and $B_{i}=\int \rho_{B_{i}}(z) d z \Phi(z, \mathbf{k}, \omega)$ gives a homogeneous system of linear equations. Equating the determinant of this linear system to zero leads to the dispersion expressing the frequency through the wave vector.

\subsection{Estimation of wave retardation in double-wall nanotubes and bilayer graphene}

The dispersion equation (57) has the simple analytical solution:

$$
\omega^{2}=\frac{\omega_{1}^{(0) 2}+\omega_{2}^{(0) 2}}{2} \pm \sqrt{\left[\frac{\omega_{1}^{(0) 2}-\omega_{2}^{(0) 2}}{2}\right]^{2}+F^{2}} .
$$

Here $\omega_{i}^{(0) 2}=\frac{\omega_{L i}^{2}\left(m^{2}+k^{2} R_{i}^{2}\right) I_{m}\left(k R_{i}\right) K_{m}\left(k R_{i}\right)}{R_{i}}$,

$$
F^{2}=\frac{\omega_{L 2}^{2} \omega_{L 1}^{2}\left(m^{2}+k^{2} R_{2}^{2}\right)\left(m^{2}+k^{2} R_{1}^{2}\right)}{R_{1} R_{2}} K_{m}\left(k R_{2}\right) K_{m}\left(k R_{2}\right) I_{m}\left(k R_{1}\right) I_{m}\left(k R_{1}\right),
$$

$\omega_{L i}^{2}=4 \pi e^{2} n_{e i} / m$ is surface Langmuir frequency squared and $n_{e i}$ is the surface electron density. It can be seen that in the case when $k\left(R_{2}-R_{1}\right) \gg 1$ (i.e. the wavelength is less than distance between walls), the walls oscillate independently. In the opposite case, oscillations are coupled strongly and one of the oscillation frequencies is anomalously small. In the nonclassical case of the Fermi-Dirac distribution for electrons, solutions can be obtained by using numerical methods.

The behavior of frequencies is demonstrated in Fig. 3. One branch corresponds to the mode with larger frequency which is proportional to the sum of frequencies of the single walls. Another branch with considerably smaller frequency corresponds to the mode with the frequency which is proportional to the difference of frequencies of single walls. The wave phase velocity is defined as $v_{p h}=\omega / k$. Therefore, the phase velocity corresponding to the mode with the smaller frequency is considerably small. Figure 4 demonstrates the possibility of slowing the wave down by more than a factor of 250 in bilayer graphene. The condition of Cerenkov synchronism can be fulfilled for $\pi$-electron at such retardation. 


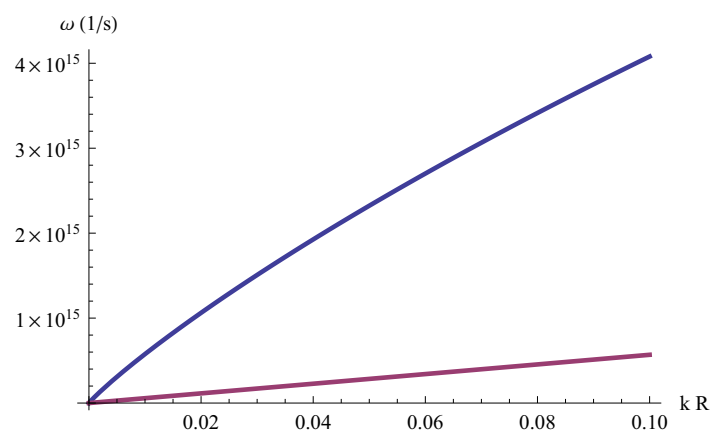

Fig. 3. Dependence of frequencies on wave vector $k$ for double-wall nanotube.

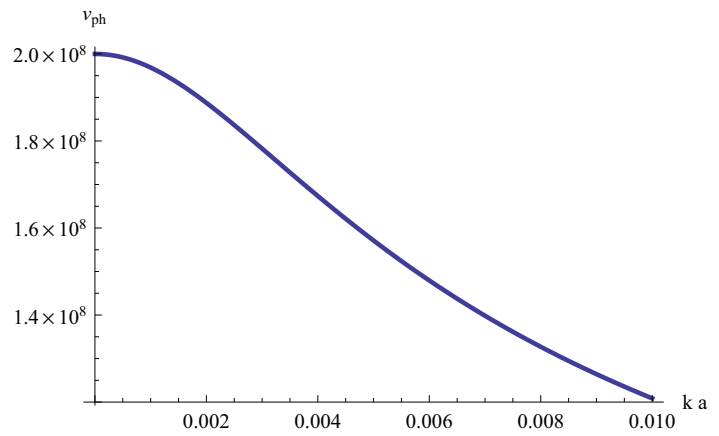

Fig. 4. Dependence of phase velocity $v_{p h}$ on wave vector $k$ for bilayer graphene.

\section{QUASI-METALLIC CARBON NANOTUBES AS TERAHERTZ EMITTERS}

The next scheme of $\mathrm{THz}$ generation $[42,43]$ is based on the electric-field induced heating of the electron gas in a SWNT resulting in the population inversion of optically active states with energy difference within the $\mathrm{THz}$ spectrum. It is well known that the elastic backscattering processes in metallic SWNTs are strongly suppressed [44], and in a high enough electric field charge carriers can be accelerated up to the energy allowing emission of optical/zone-boundary phonons. At this energy, corresponding to a frequency of about $40 \mathrm{THz}$, the major scattering mechanism switches on abruptly resulting in current saturation [45-49]. As will be shown hereafter, for certain types of carbon nanotubes the heating of electrons to the energies below the phonon-emission threshold results in the spontaneous $\mathrm{THz}$ emission with the peak frequency controlled by an applied voltage.

The electron energy spectrum $\mathcal{E}(k)$ of a metallic SWNT in the vicinity of the Fermi energy linearly depends on the electron wave vector $k$ and has the form $\varepsilon(k)= \pm \hbar v_{F}\left|k-k_{0}\right|$, where $v_{F} \approx 9.8 \times 10^{5} \mathrm{~m} / \mathrm{s}$ is the Fermi velocity of graphene, which corresponds to the commonly used tight-binding matrix element $\gamma_{0}=3.033 \mathrm{eV}[3,4]$. Herein the zero of energy is defined as the Fermi energy position in the absence of an external $\mathrm{f}$ eld. When the voltage, $V$, is applied between the SWNT ends, the electron distribution is shifted in the way shown by the heavy lines in Fig. 5(a) corresponding to the filled electron states. This shift results in inversion of population and, correspondingly, in optical transitions between filled states in the conduction band and empty states in the valence band. The spectrum of optical transitions is determined by the distribution function for hot carriers, which in turn depends on the applied voltage and scattering processes in the SWNT. It is well known that the major scattering mechanism in SWNTs is due to electron-phonon interaction [45-47,49]. Since the scattering processes erode the inversion of electron population, an optimal condition for observing the discussed optical 

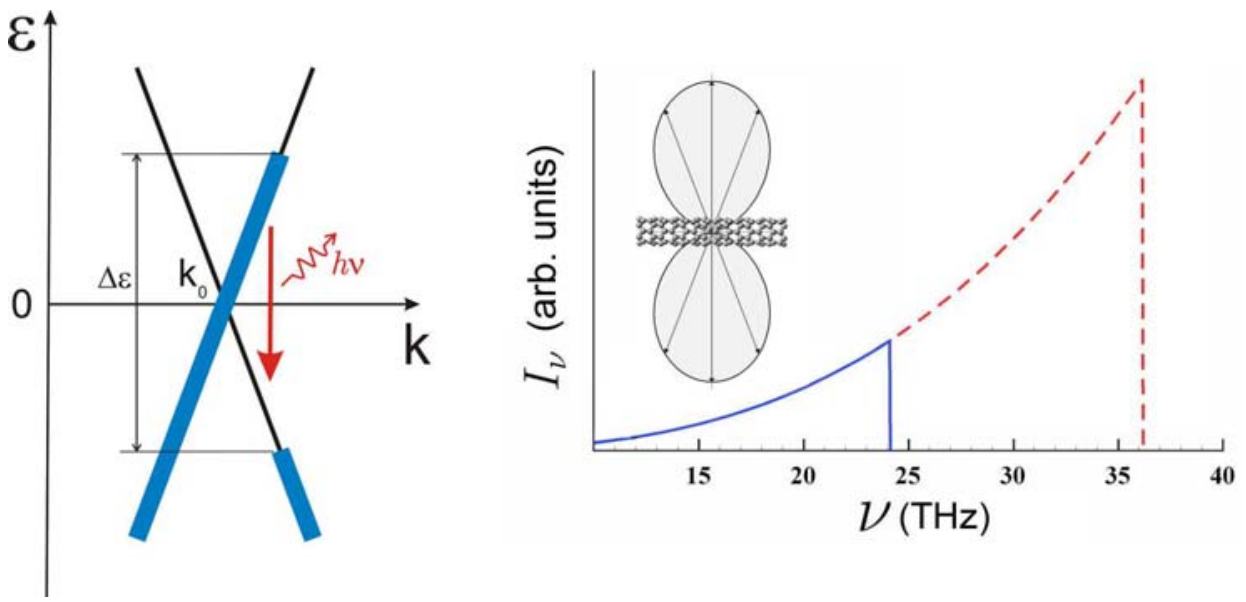

Fig. 5. (a) The scheme of THz photon generation by hot carriers in quasi-metallic SWNTs. (b) The spectral density of spontaneous emission as a function of frequency for two values of applied voltage: solid line for $V=0.1 \mathrm{~V}$; dashed line for $V=0.15 \mathrm{~V}$. The inset shows the directional radiation pattern of the $\mathrm{THz}$ emission with respect to the nanotube axis.

transitions is when the length of the SWNT $L<l_{a c}$, where the electron mean-free path for acoustic phonon scattering is $l_{a c} \approx 2 \mu \mathrm{m}$ [47]. Below, only such short SWNTs with ideal Ohmic contacts [46] are considered in the ballistic transport regime, when the energy acquired by the electron along the length of the tube, $\Delta \varepsilon=e V$, does not exceed the value of $\hbar \Omega=$ $0.16 \mathrm{eV}$ at which the fast emission of high-energy phonons begins [47]. In this so-called lowbias regime [45-47], in which the current in the nanotube is given by the Büttiker-Landauer-type formula, $I \approx\left(4 e^{2} / h\right) V$, the distribution function of hot electrons is

$$
f_{e}(k)=\left\{\begin{array}{ll}
1, & 0<k-k_{0}<\Delta \varepsilon / 2 \hbar v_{F} \\
0, & k-k_{0}>\Delta \varepsilon / 2 \hbar v_{F}
\end{array} .\right.
$$

The distribution function for hot holes, $f_{h}(k)$, has the same form as $f_{e}(k)$.

Let us select a SWNT with the crystal structure most suitable for observation of the discussed effect. First, the required nanotube should have metallic conductivity and, second, the optical transitions between the lowest conduction subband and the top valence subband should be allowed. SWNTs with true metallic energy band structure, for which the energy gap is absent for any SWNT radius, are armchair $(n, n)$ SWNTs only [4, 50-53]. However, for armchair SWNTs the optical transitions between the first conduction and valence subbands are forbidden $[54,55]$. So for the observation of $\mathrm{THz}$ generation it is possible to use so-called quasimetallic $(n, m)$ SWNTs with $n-m=3 p$, where $p$ is a non-zero integer. These nanotubes, which are gapless within the frame of a simple zone-folding model of the $\pi$-electron graphene spectrum [3], are in fact narrow-gap semiconductors due to curvature effects. Their bandgap is given by $\varepsilon_{g}=\hbar v_{F} b \cos 3 \theta /\left(8 R_{c n}^{2}\right)[50,53]$, where $b=1.42 \AA$ is the nearest-neighbor distance between two carbon atoms and $\theta=\arctan [\sqrt{3} m /(2 n+m)]$ is the chiral angle [3]. It can be seen from the expression for $\varepsilon_{g}$ that the gap is decreasing rapidly with increasing nanotube radius. For large values of $R_{c n}$ this gap can be neglected even in the case of moderate applied voltages due to Zener tunneling of electrons across the gap. It is easy to show in a fashion similar to Zener's original work [56] that the tunneling probability in quasi-metallic SWNTs is given by $\exp \left(-\alpha \varepsilon_{g}^{2} / e E \hbar v_{F}\right)$, where $\alpha$ is a numerical factor close to unity*. For example,

${ }^{*}$ For the energy spectrum near the band edge given by $\varepsilon= \pm\left[\varepsilon_{g}^{2} / 4+\hbar^{2} v_{F}^{2}\left(k-k_{0}\right)^{2}\right]^{1 / 2}$, it can be 
for a zigzag $(30,0)$ SWNT the gap is $\varepsilon_{g} \approx 6 \mathrm{meV}$ and the Zener breakdown takes place in the electric field $E \sim 10^{-1} \mathrm{~V} / \mu \mathrm{m}$. Since almost the whole voltage drop in the ballistic regime occurs within the few-nanometer regions near the contacts [57] a typical bias voltage of $0.1 \mathrm{~V}$ corresponds to an electric field, which is more than sufficient to achieve a complete breakdown. In what follows all calculations are performed for a zigzag $(3 p, 0)$ SWNT of large enough radius $R_{c n}$ and for applied voltages exceeding the Zener breakdown, so that the finite-gap effects can be neglected. The obtained results can be easily generalized for any quasi-metallic large-radius SWNT.

Optical transitions in SWNTs have been the subject of extensive research [54, 55, 58-62]. Let us treat these transitions using the results of the nearest-neighbor orthogonal $\pi$-electron tight binding model [3]. Despite its apparent simplicity and well known limitations, this model has been extremely successful in describing low-energy optical spectra and the electronic properties of SWNTs [63]. The main goal is to calculate the spectral density of spontaneous emission, $I_{\nu}$, which is the probability of optical transitions per unit time for the photon frequencies in the interval $(\nu, \nu+d \nu)$ divided by $d \nu$. In the dipole approximation [38] this spectral density is given by

$$
I_{\nu}=\frac{8 \pi e^{2} \nu}{3 c^{3}} \sum_{i, f} f_{e}\left(k_{i}\right) f_{h}\left(k_{f}\right)\left|\left\langle\Psi_{f}\left|\hat{v}_{z}\right| \Psi_{i}\right\rangle\right|^{2} \delta\left(\varepsilon_{i}-\varepsilon_{f}-h \nu\right) .
$$

Equation (66) contains the matrix element of the electron velocity operator. In the frame of the tight binding model, this matrix element for optical transitions between the lowest conduction and the highest valence subbands of the $(3 p, 0)$ zigzag SWNT can be written as $[55,58]$

$$
\left\langle\Psi_{f}\left|\hat{v}_{z}\right| \Psi_{i}\right\rangle=\frac{b \omega_{i f}}{8} \delta_{k_{f}, k_{i}}
$$

where $\hbar \omega_{i f}=\varepsilon_{i}-\varepsilon_{f}$ is the energy difference between the initial $(i)$ and the final $(f)$ states. These transitions are associated with the light polarized along the nanotube axis $z$, in agreement with the general selection rules for SWNTs [54]. Substituting Eq. (67) in Eq. (66) and performing necessary summation, we get

$$
I_{\nu}=L f_{e}\left(\pi \nu / v_{F}\right) f_{h}\left(\pi \nu / v_{F}\right) \frac{\pi^{2} e^{2} b^{2} \nu^{3}}{6 c^{3} \hbar v_{F}}
$$

Equation (68) has broader applicability limits than the considered case of $L<l_{a c}$ and $e V<$ $\hbar \Omega$, in which the distribution functions for electrons and holes are given by Eq. (65). In the general case there is a strong dependence of $I_{\nu}$ on the distribution functions, which have to be calculated taking into account all the relevant scattering mechanisms [45-47,49]. In the discussed ballistic regime the spectral density has a universal dependence on the applied voltage and photon frequency for all quasi-metallic SWNTs. In Fig. 5(b) the spectral density is shown for two values of the voltage. It is clearly seen that the maximum of the spectral density of emission has strong voltage dependence and lies in the $\mathrm{THz}$ frequency range for experimentally attainable voltages. The directional radiation pattern, shown in the inset of Fig. 5(b), reflects the fact that emission of light polarized normally to the nanotube axis is forbidden by the selection rules for the optical transitions between the lowest conduction subband and the top valence subband.

For some device applications it might be desirable to emit photons propagating along the nanotube axis, which is possible in optical transitions between the SWNT subbands characterized by angular momenta differing by one $[4,54]$. To achieve the emission of these photons by the electron heating, it is necessary to have an intersection of such subbands within the energy range accessible to electrons accelerated by attainable voltages. From the analysis of different

shown that $\alpha=\pi / 4$. 
types of SWNTs, it follows that the intersection is possible, e.g., for the lowest conduction subbands in several semiconducting zigzag nanotubes and in all armchair nanotubes. However, for an effective $\mathrm{THz}$ emission from these nanotubes it is necessary to move the Fermi level very close to the subband intersection point [42]. Therefore, obtaining the THz emission propagating along the nanotube axis is a more difficult technological problem than generating emission shown in Fig. 5(b).

\section{CHIRAL CARBON NANOTUBES AS FREQUENCY MULTIPLIERS}

Another proposal for using SWNTs for THz applications [64, 65] is based on chiral nanotubes, which represent natural superlattices. For example, a $(10,9)$ single-wall nanotube has a radius which differs from the radius of the most commonly studied $(10,10)$ nanotube by less than five percent, whereas the translational period $T$ along the axis of the $(10,9)$ SWNT is almost thirty times larger than the period of the $(10,10)$ nanotube. Correspondingly, the first Brillouin zone of the $(10,9)$ nanotube is thirty times smaller than the first zone for the $(10,10)$ tube. However such a Brillouin zone reduction cannot influence electronic transport unless there is a gap opening between the energy subbands resulting from the folding of graphene spectrum. It can be shown that an electric field normal to the nanotube axis opens noticeable gaps at the edge of the reduced Brillouin zone, thus turning a long-period nanotube of certain chirality into a 'real' superlattice. This gap opening is a general property of chiral nanostructures exposed to a transverse electric field [66-68]. The field-induced gaps are most pronounced in $(n, 1)$ SWNTs $[65,69]$. Figure 6(a) shows the opening of an electric-field induced gap near the edge

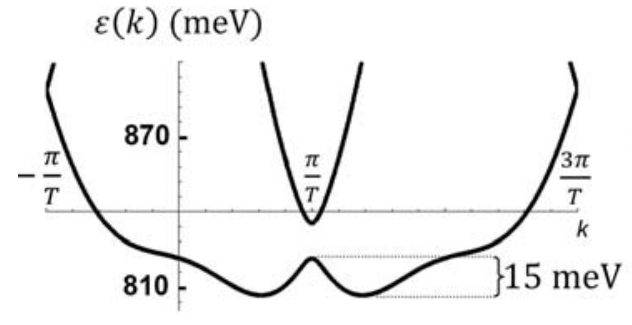

(a)

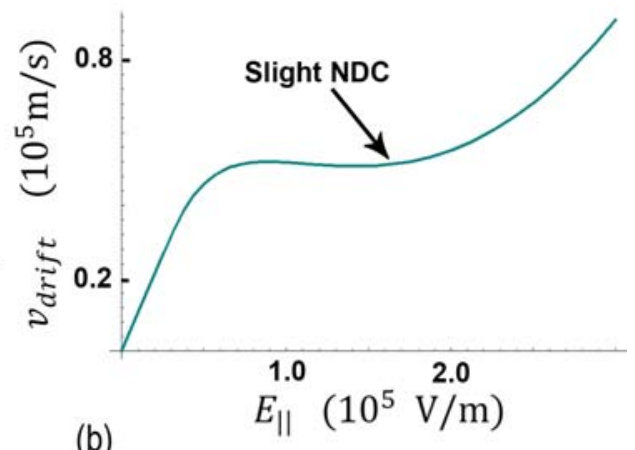

(b)

Fig. 6. (a) Energy spectrum of the $(6,1) \mathrm{SWNT}$ in a transverse electric field, $E_{\perp}=4 \mathrm{~V} / \mathrm{nm}$. (b) The electron drift velocity in the lowest conduction subband of a $(6,1)$ SWNT as a function of the longitudinal electric field, in the presence of acoustic-phonon scattering.

of the Brillouin zone of a $(6,1)$ SWNT. This gap opening results in the appearance of a negative effective-mass region in the nanotube energy spectrum. The typical electron energy in this part of the spectrum of $15 \mathrm{meV}$ is well below the optical phonon energy $\hbar \Omega \approx 160 \mathrm{meV}$, so that it can be easily accessed in moderate heating electric fields. The negative effective mass results in the negative differential conductivity (NDC), as can be seen from Fig. 6(b). The NDC characteristic presented in Fig. 6(b) is calculated assuming the energy-independent scattering time $\tau=1$ ps. However, when the carrier energy reaches the optical or edge-phonon energy, the scattering time $\tau$ increases abruptly. This results in more pronounced NDC, which can be used for generating electromagnetic radiation in the $\mathrm{THz}$ range.

The effect of the negative effective mass in chiral nanotubes [65], not only results in the NDC but also leads to an efficient frequency multiplication in the THz range. The results of calculations of the electron velocity in the presence of the time dependent longitudinal electric 


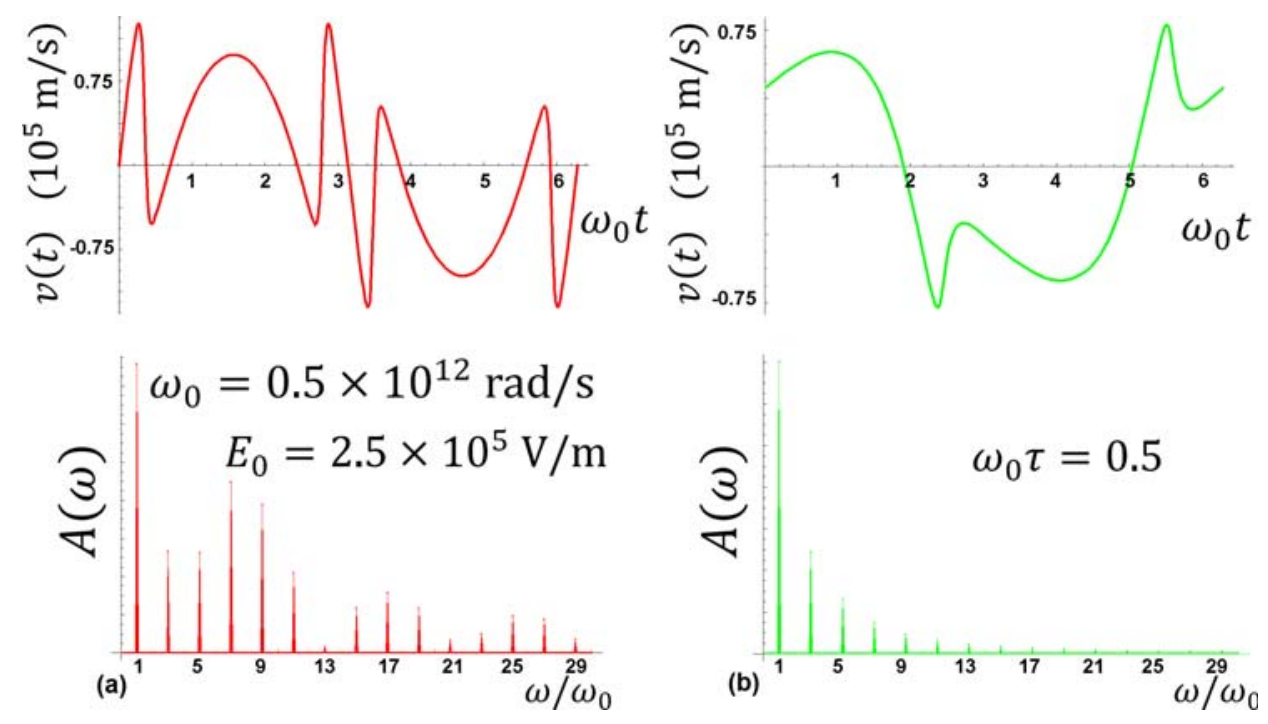

Fig. 7. Time dependence of the electron velocity in the lowest conduction subband of a $(6,1)$ SWNT under the inf uence of a pump harmonic longitudinal electric field, $E_{\|}(t)=$ $E_{0} \sin \left(\omega_{0} t\right)$, and its correspondent spectral distribution $A(\omega)$ : (a) in the ballistic transport regime; (b) in the presence of scattering with the relaxation time $\tau=10^{-12} \mathrm{~s}$.

field are presented in Fig. 7. One of the advantages of a frequency multiplier based on chiral SWNTs, in comparison with the conventional superlattices [70], is that the dispersion relation in such a system can be controlled by the transverse electric field $E_{\perp}$.

\section{ARMCHAIR NANOTUBES IN A MAGNETIC FIELD AS TUNABLE THZ DETECTORS AND EMITTERS}

The problem of detecting THz radiation is known to be at least as challenging as creating reliable THz sources. The proposal of a novel detector $[64,65]$ is based on several features of truly gapless (armchair) SWNTs. It should be noted that the electron energy spectrum of SWNTs depends crucially on the magnetic field [71-74]. The main property to be utilised is opening of a band gap in these SWNTs in a magnetic field along the nanotube axis $[3,4]$. For a $(10,10)$ SWNT this gap corresponds to approximately $1.6 \mathrm{THz}$ in the field of $10 \mathrm{~T}$. For attainable magnetic fields, the gap grows linearly with increasing both magnetic field and the nanotube radius. It can be shown [65] that the same magnetic field also allows dipole optical transitions between the top valence subband and the lowest conduction subband, which are strictly forbidden in armchair SWNTs without the field [54].

In Fig. 8 it is shown how the energy spectrum and matrix elements of the dipole optical transitions polarized along the nanotube axis are modified in the presence of a longitudinal magnetic field. In the frame of the nearest-neighbor tight binding model, one can show that for a $(n, n)$ armchair nanotube the squared matrix element of the velocity operator between the states at the edge of the gap opened by the magnetic field is given by a simple analytic expression:

$$
\left|\left\langle\Psi_{n}^{v}\left|\hat{v}_{z}\right| \Psi_{i}^{c}\right\rangle\right|^{2}=\frac{4}{3}\left[1-\frac{1}{4} \cos ^{2}\left(\frac{f}{n} \pi\right)\right] v_{F}^{2},
$$

where $f=e B R^{2} /(2 \hbar)$. For experimentally attainable magnetic fields, when the magnetic flux through the SWNT is much smaller than the flux quantum, the absolute value of the velocity 


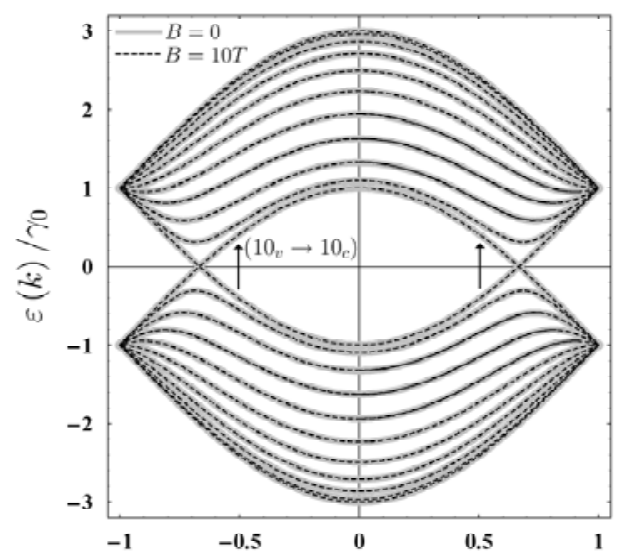

(a)

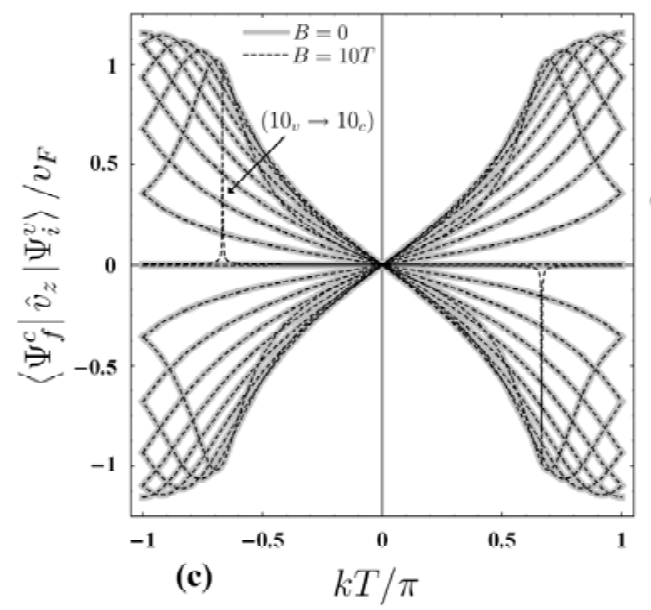

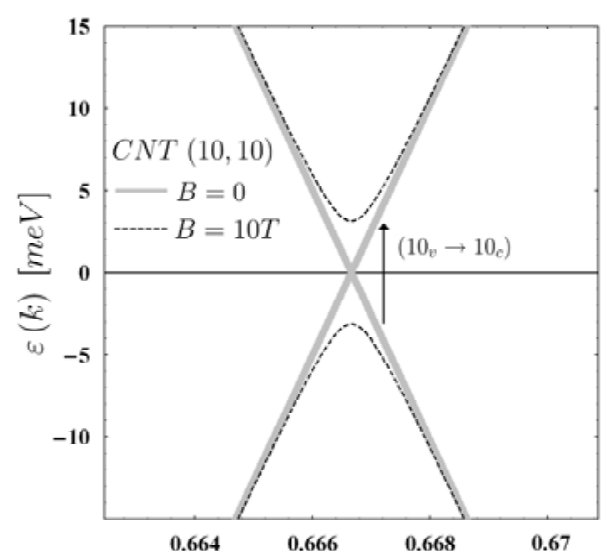

(b)

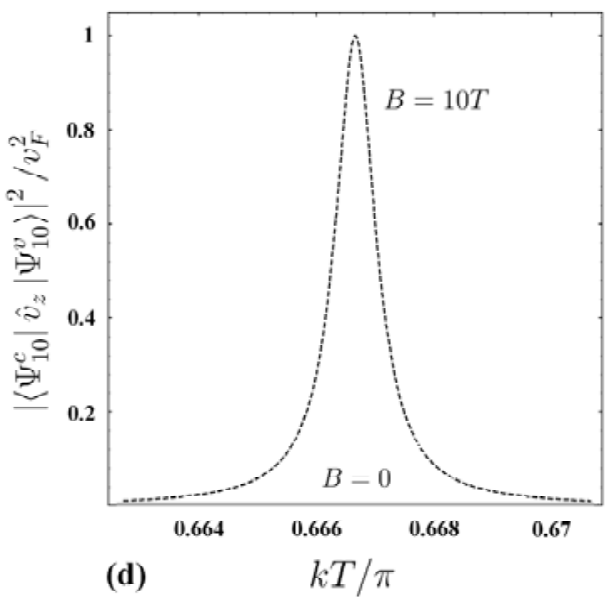

Fig. 8. (a) Band structure of a $(10,10)$ nanotube, with and without an external magnetic field along the nanotube axis. (b) Detailed view of the gap, which is opened between the top valence subband and the lowest conduction subband in an external field $B=10 \mathrm{~T}$. (c) The change in the dipole optical transitions matrix elements, for light polarized along the SWNT axis, due to the introduction of the external magnetic field. The only appreciable change is in the appearance of a high narrow peak associated with the transition $\left(10_{v} \rightarrow 10_{c}\right)$, which is not allowed in the absence of the magnetic field. Herein the energy subbands are numbered in the same way as in Ref. 3. (d) Dependence of the squared dipole matrix element for the transition $\left(10_{v} \rightarrow 10_{c}\right)$ on the 1D wave vector $k$, with and without an external magnetic field.

operator is close to $v_{F}$. Equation (69) is relevant to the transitions between the highest valence subband and the lowest conduction subband only for $f \leq 1 / 2$, since for the higher values of $f$ the order of the nanotube subbands is changed. Notably, the same equation allows one to obtain the maximum value of the velocity operator in any armchair SWNT for the transitions polarized along its axis: this value cannot exceed $2 v_{F} / \sqrt{3}$ (see panel (c) in Fig. 8).

The electron (hole) energy spectrum near the bottom (top) of the band gap produced by the magnetic field is parabolic as a function of a carrier momentum along the nanotube axis. This dispersion results in a Van Hove singularity in the reduced density of states, which in turn leads 
to a very sharp absorption maximum near the band edge and, correspondingly, to a very high sensitivity of the photocurrent to the photon frequency, see Fig. 9.
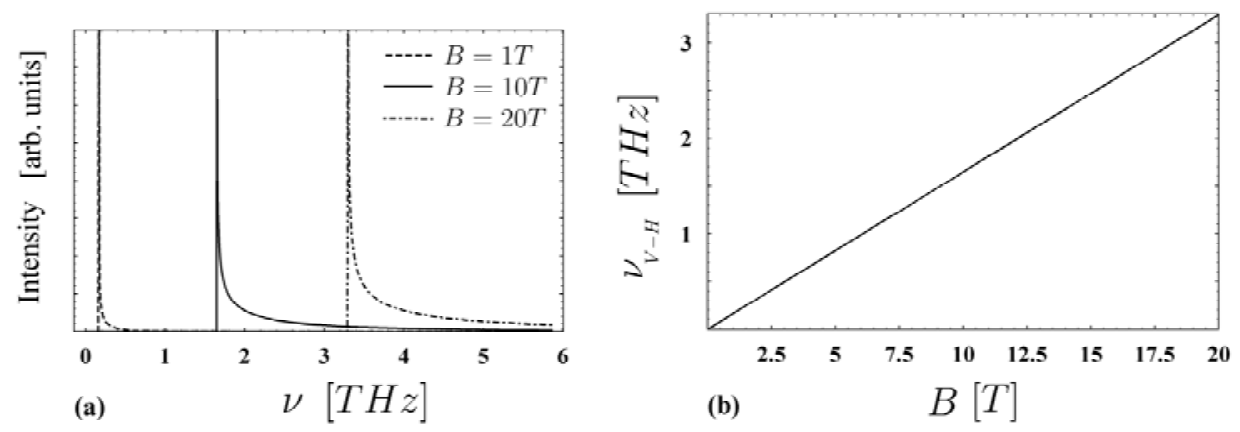

Fig. 9. (a) Calculated photon absorption spectra for a $(10,10)$ SWNT, for three different magnetic field values. The absorption intensity is proportional to the product of $\left|\left\langle\Psi_{10}^{v}\left|\hat{v}_{z}\right| \Psi_{10}^{c}\right\rangle\right|^{2}$ and the joint density of states. (b) Dependence of the position of the peak in the absorption intensity, associated with the Van Hove singularity, on the magnetic field strength.

Notably, the same effect can be used for the generation of a very narrow emission line having the peak frequency tunable by the applied magnetic field. Population inversion can be achieved, for example, by optical pumping with light polarized normally to the nanotube axis, as shown in Fig. 10.
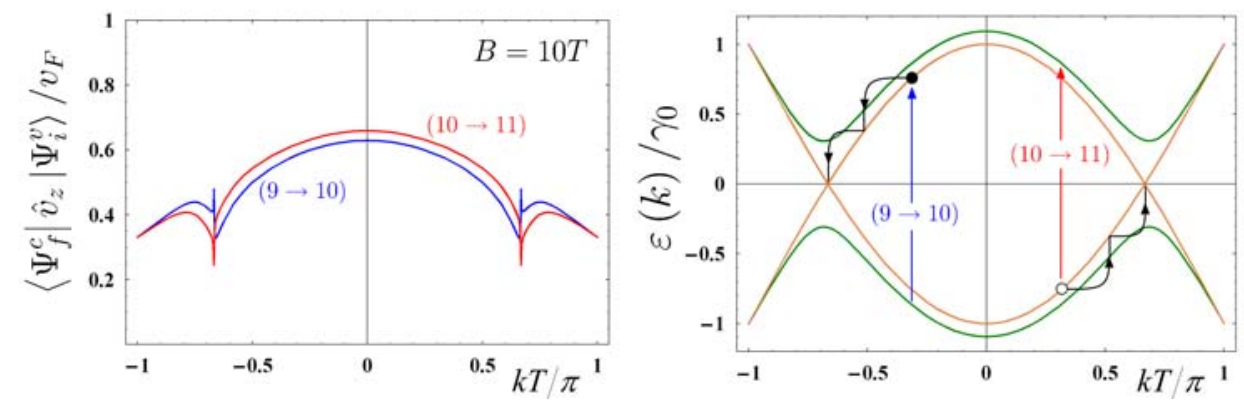

Fig. 10. A scheme for creating population inversion between the lowest conduction subband and the top valence subband of an armchair SWNT in a magnetic field. The left plot shows the calculated matrix elements of the relevant dipole optical transitions polarized normally to the axis of a $(10,10)$ SWNT. The right plot shows several energy subbands closest to the Fermi level and illustrates the creation of photoexcited carriers and their non-radiative thermalization.

\section{CONCLUSION}

In this paper we have demonstrated several schemes for emitting and detecting terahertz radiation by CNTs.

One of the ideas exploits an analogy between CNTs and macroscopic traveling wave tube. Strong slowing down of surface electromagnetic waves in SWNTs and the ballisticity of the electron motion over typical CNTs length allows them to be used as nanoscale Cerenkov-type emitters in the terahertz frequency range. The use of SWNT bundles instead of an isolated SWNT is suggested as a means to decrease the threshold current density. 
We also have demonstrated that a quasi-metallic SWNT can emit THz radiation when a potential difference is applied to its ends. The typically required voltages and nanotube parameters are similar to those available in the state-of-the-art transport experiments. The maximum of the spectral density of emission is shown to have strong voltage dependence, which is universal for all quasi-metallic SWNTs in the ballistic regime. Therefore, the discussed effect can be used for creating a THz source with frequency controlled by the applied voltage. Appropriately arranged arrays of nanotubes should be considered as promising candidates for active elements of amplif ers and generators of coherent $\mathrm{THz}$ radiation.

We have also shown that an electric field, which is applied normally to the axis of longperiod chiral nanotubes, significantly modifies their band structure near the edge of the Brillouin zone. This results in a negative effective-mass region at an energy scale below the high-energy phonon-emission threshold. This effect can be used for efficient frequency multiplication in the $\mathrm{THz}$ range. Finally, we have discussed the feasibility of using a magnetic field, which opens energy gaps and allows optical transitions in armchair nanotubes, for creating tunable $\mathrm{THz}$ detectors and emitters.

\section{Acknowledgements}

The research was partially supported by the EU FP7 projects TerACaN FP7-230778 and CACOMEL FP7-247007, ISTC-project B-1708, RFBR (Russia) projects 10-02-00077 and 10-0290001, BRFBR (Belarus) project F08R-009, 'Development of Scientific Potential of Russian

Higher Education' Program (project 2.1.2/2115), Federal goal-oriented program 'Scientific and scientific-educational personnel of innovative Russia'.

\section{References}

[1] M. Lee and M. C. Wanke, "Searching for a solid-state terahertz technology," Science 316, 64-65 (2007). [doi:10.1126/science.1141012].

[2] S. Iijima, "Helical microtubules of graphitic carbon," Nature 354, 56-58 (1991). [doi: $10.1038 / 354056 \mathrm{a} 0$ Letter].

[3] R. Saito, G. Dresselhaus, and M. S. Dresselhaus, Physical Properties of Carbon Nanotubes, Imperial College Press, London (1998).

[4] S. Reich, C. Thomsen, and J. Maultzsch, Carbon Nanotubes: Basic Concepts and Physical Properties, Wiley, Berlin (2004).

[5] D. Dragoman and M. Dragoman, “Terahertz fields and applications," Prog. Quantum Electron. 28, 1-66 (2004). [doi:10.1016/S0079-6727(03)00058-2].

[6] D. Dragoman and M. Dragoman, "Terahertz oscillations in semiconducting carbon nanotube resonant-tunnelling diodes," Physica E 24, 282-289 (2004). [doi:10.1016/j.physe.2004.05.001].

[7] D. Dragoman and M. Dragoman, "Terahertz continuous wave amplification in semiconductor carbon nanotubes," Physica E 25, 492-496 (2005). [doi:10.1016/j.physe.2004.08.001].

[8] K. G. Batrakov, P. P. Kuzhir, and S. A. Maksimenko, "Radiative instability of electron beam in carbon nanotubes," Proc. SPIE) 6328, 632801 (2006). [doi:10.1117/12.678029].

[9] K. G. Batrakov, P. P. Kuzhir, S. A. Maksimenko, and C. Tomsen, "Carbon nanotube as a Cherenkov-type light emitter and free electron laser," Phys. Rev. B 79, 125408-12420 (2009). [doi:10.1103/PhysRevB.79.125408].

[10] K. G. Batrakov, P. P. Kuzhir, and S. A. Maksimenko, "Toward the nano-fel: Undulator and Cherenkov mechanisms of light emission in carbon nanotubes," Physica E 40, 1065-1068 (2008). [doi:10.1016/j.physe.2007.08.003]. 
[11] K. G. Batrakov, P. P. Kuzhir, and S. A. Maksimenko, "Stimulated emission of electron beam in nanotube bundles," Physica E 40, 2370-2374 (2008). [doi:10.1016/j.physe.2007.07.029].

[12] P. P. Kuzhir, K. G. Batrakov, and S. A. Maksimenko, "Generation and propagation of electromagnetic waves in carbon nanotubes: New propositon for optoelectronics and biomedical applications," Synthesis and Reactivity in Inorganic, Metal-Organic and NanoMetal Chemistry 37, 341-346 (2007). [doi:10.1080/15533170701392420].

[13] K. G. Batrakov, P. P. Kuzhir, and S. A. Maksimenko, "Electromagnetic wave slowing down in graphene bilayer," Proceedings of the 2nd International Workshop, Bad Honnef, Germany 28-30 October 2009, 40-42 (2009).

[14] R. Varian and S.Varian, "A high frequency amplifier and oscillator," J. Appl. Phys. 10, 321-328 (1939). [doi:10.1063/1.1707311].

[15] R. Kompfner, The Invention of the Travelling Wave Tube, San Francisco Press, San Francisco (1964).

[16] J. M. J. Madey, "Stimulated emission of bremsstrahlung in a periodic magnetic f eld," J. Appl. Phys. 42, 1906-1914 (1971). [doi:10.1063/1.1660466].

[17] F. A. Hopf, P. Meystre, M. O. Scully, and W. H. Louisell, "Classical theory of a free-electron laser," Phys. Rev. Lett. 37, 1215-1218 (1976). [doi:10.1103/PhysRevLett.37.1215].

[18] T. C. Marshall, Free Electron Lasers, Macmillan, New York (1985).

[19] E. L. Saldin, E. A. Schneidmiller, and M. V. Yurkov, The Physics of Free Electron Lasers, Springer, Berlin (2000).

[20] "Slac linac coherent light source (1cls) conceptual design report," JSLAC-R-593 (2002).

[21] M. Altarelli, "Xfel: The european x-ray free- electron laser. technical design report," Preprint DESY 2006-097, see also http://xfel.desy.de (2006).

[22] S. J. Smith and E. M. Purcell, "Visible light from localized surface charges moving across a grating," Phys. Rev. 92, 1069-1069 (1953). [doi:10.1103/PhysRev.92.1069].

[23] V. Baryshevsky, K. Batrakov, and I. Dubovskaya, "Parametric (quasi-cerenkov) x-ray free electron lasers," J. Phys. D: Applied Physics 24, 1250-1257 (1991). [doi:10.1088/00223727/24/8/005].

[24] R. Q. Twiss, "Radiation transfer and the possibility of negative absorption in radio astronomy," Austral. J. Phys. 11, 564-579 (1958).

[25] J. Schneider, "Stimulated emission of radiation by relativistic electrons in a magnetic field," Phys. Rev. Lett. 2, 504-505 (1959). [doi:10.1103/PhysRevLett.2.504].

[26] V. G. Baryshevsky and I. D. Feranchuk, "Parametric beam instability of relativistic charged particles in a crystal," Phys. Lett. A 102, 141-144 (1984). [doi:10.1016/03759601(84)90799-0].

[27] A. Friedman, A. Gover, G. Kurizki, S. Ruschin, and A. Yariv, "Spontaneous and stimulated emission from quasifree electrons," Rev. Mod. Phys. 60, 471-535 (1988). [doi:10.1103/RevModPhys.60.471].

[28] R. B. Miller, An Introduction to the Physics of Intense Charged Psrticles Beams, Plenum Press, New York (1982).

[29] S. Frank, P. Poncharal, Z. L. Wang, and W. A. de Heer, "Carbon nanotube quantum resistors," Science 280, 1744-1746 (1998). [doi:10.1126/science.280.5370.1744].

[30] C. Berger, Y. Yi, Z. L. Wang, and W. A. de Heer, "Multiwalled carbon nanotubes are ballistic conductors at room temperature," Appl. Phys. A 74, 363-365 (2002). [doi:10.1007/s003390201279].

[31] C. Berger, P. Poncharal, Y. Yi, and W. A. de Heer, "Ballistic conduction in multiwalled carbon nanotubes,” J. Nanosci. Nanotechn. 3, 171-177 (2003). [doi:10.1166/jnn.2003.180]. 
[32] Z. Yao, C. L. Kane, and C. Dekker, "High-field electrical transport in single-wall carbon nanotubes," Phys. Rev. Lett. 84, 2941-2944 (2000). [doi:10.1103/PhysRevLett.84.2941].

[33] B. Q. Wei, R. Vajtai, and P. M. Ajayan, "Reliability and current carrying capacity of carbon nanotubes," Appl. Phys. Lett. 79, 1172-1174 (2001). [doi:10.1063/1.1396632].

[34] R. Vajtai, B. Q. Wei, Z. J. Zhang, Y. Jung, G. Ramanath, and P. M. Ajayan, "Building carbon nanotubes and their smart architectures," Smart Mater. Struc. 11, 691-699 (2002). [doi:10.1088/0964-1726/11/5/311].

[35] G. Y. Slepyan, S. A. Maksimenko, A. Lakhtakia, O. Yevtushenko, and A. V. Gusakov, "Electrodynamics of carbon nanotubes: Dynamic conductivity, impedance boundary conditions, and surface wave propagation," Phys. Rev. B 60, 17136-17149 (1999). [doi:10.1103/PhysRevB.60.17136].

[36] edited by A. Lakhtakia, The Handbook of Nanotechnology: Nanometer Structure Theory, Modeling, and Simulation, SPIE Press, Bellingham (2004).

[37] M. S. Dresselhaus, G. Dresselhaus, and P. Avouris, Carbon Nanotubes, Springer, Berlin (2001).

[38] V. B. Berestetskii, E. M. Lifshitz, and L. P. Pitaevskii, Quantum Electrodynamics, Butterworth-Heinemann, Oxford (1997).

[39] L. D. Landau and E. M. Lifshitz, Quantum Mechanics: Non-Relativistic Theory, Pergamon Press, Oxford (1977).

[40] E. M. Lifshitz and L. P. Pitaevskii, Physical Kinetics, Pergamon Press, Oxford (1981).

[41] O. Zvelto, Principles of Lasers, Plenum, New York (2004).

[42] O. V. Kibis and M. E. Portnoi, "Carbon nanotubes: A new type of emitter in the terahertz range,” Tech. Phys. Lett. 31, 671-673 (2005). [doi:10.1134/1.2035361].

[43] O. V. Kibis, M. R. da Costa, and M. E. Portnoi, "Generation of terahertz radiation by hot electrons in carbon nanotubes," Nano Lett. 7, 3414-3417 (2007). [doi: $10.1021 / \mathrm{n} 10718418$ ].

[44] T. Ando, T. Nakanishi, and R. Saito, "Impurity scattering in carbon nanotubes - absence of back scattering,” J. Phys. Soc. Jpn. 67, 1704-1713 (1997). [doi:10.1143/JPSJ.67.1704].

[45] Z. Yao, C. Kane, and C. Dekker, "High-field electrical transport in single-wall carbon nanotubes," Phys. Rev. Lett. 84, 2941-2944 (2000). [doi:10.1103/PhysRevLett.84.2941].

[46] A. Javey, J. Guo, M. Paulsson, Q. Wang, D. Mann, M. Lundstrom, and H. Dai, "High-f eld quasiballistic transport in short carbon nanotubes," Phys. Rev. Lett. 92, 106804 (2004). [doi:10.1103/PhysRevLett.92.106804].

[47] J.-Y. Park, S. Resenblatt, Y. Yaish, V. Sazonova, H. Ustunel, S. Braig, T. Arias, P. Brouwer, and P. McEuen, "Electron-phonon scattering in metallic single-wall carbon nanotubes," Nano Lett. 4, 517-520 (2004). [doi:10.1021/n1035258c].

[48] M. Freitag, V. Pereibenos, J. Chen, A. Stein, J. Tsang, J. Misewich, R. Martel, and P. Avouris, "Hot carrier electroluminescence from a single carbon nanotube," Nano Lett. 4, 1063-1066 (2004). [doi:10.1021/n1049607u].

[49] V. Perebeinos, J. Tersoff, and P. Avouris, "Electron-phonon interaction and transport in semiconducting carbon nanotubes," Phys. Rev. Lett. 94, 086802 (2005). [doi:10.1103/PhysRevLett.94.086802].

[50] C. L. Kane and E. J. Mele, "Size, shape, and low energy electronic structure of carbon nanotubes,” Phys. Rev. Lett. 78, 1932-1935 (1997). [doi:10.1103/PhysRevLett.78.1932].

[51] M. Ouyang, J.-L. Huang, C. L. Cheung, and C. M. Lieber, "Energy gaps in "metallic" single-walled carbon nanotubes," Science 292, 702-705 (2001). [doi:10.1126/science.1058853].

[52] Y. Li, U. Ravaioli, and S. V. Rotkin, "Metal-semiconductor transition and fermi velocity renormalization in metallic carbon nanotubes," Phys. Rev. B 73, 035415 (2006). [doi:10.1103/PhysRevB.73.035415]. 
[53] D. Gunlycke, C. J. Lambert, S. W. D. Bailey, D. G. Pettifor, G. A. D. Briggs, and J. H. Jefferson, "Bandgap modulation of narrow-gap carbon nanotubes in a transverse electric field,” Europhys. Lett. 73, 759-764 (2006). [doi:10.1209/epl/i2005-10446-x].

[54] I. Milošević, T. Vuković, S. Dmitrović, and M. Damnjanović, "Polarized optical absorption in carbon nanotubes: a symmetry-based approach," Phys. Rev. B 67, 165418 (2003). [doi:10.1103/PhysRevB.67.165418].

[55] J. Jiang, R. Saito, A. Grüneis, G. Dresselhaus, and M. S. Dresselhaus, "Optical absorption matrix elements in single-wall carbon nanotubes.," Carbon 42, 3169-3176 (2004). [doi:10.1016/j.carbon.2004.07.028].

[56] C. Zener, "A theory of the optical breakdown of solid dielectrics," Proc. R. Soc. Lond. 145, 523-529 (1934).

[57] A. Svizhenko and M. P. Anantram, "Effect of scattering and contacts on current and electrostatics in carbon nanotubes," Phys. Rev. B 72, 085340 (2005). [doi:10.1103/PhysRevB.72.085340].

[58] A. Grüneis, R. Saito, G. G. Samsonidze, T. Kimura, M. A. Pimenta, A. Jorio, A. G. S. Filho, G. Dresselhaus, and M. S. Dresselhaus, "Inhomogeneous optical absorption around the k point in graphite and carbon nanotubes," Phys. Rev. B 67, 165402 (2003). [doi:10.1103/PhysRevB.67.165402].

[59] V. N. Popov and L. Henrard, "Comparative study of the optical properties of single-walled carbon nanotubes within orthogonal and nonorthogonal tight-binding models," Phys. Rev. $B$ 70, 115407 (2004). [doi:10.1103/PhysRevB.70.115407].

[60] R. Saito, A. Grüneis, G. G. Samsonidze, G. Dresselhaus, M. Dresselhaus, A. Jorio, L. Cancado, M. Pimenta, and A. S. Filho, "Optical absorption of graphite and single-wall carbon nanotube," Appl. Phys. A 78, 1099-1105 (2004). [doi:10.1007/s00339-003-2459-z].

[61] S. V. Goupalov, “Optical transitions in carbon nanotubes," Phys. Rev. B 72, 195403 (2005). [doi:10.1103/PhysRevB.72.195403].

[62] Y. Oyama, R. Saito, K. Sato, J. Jiang, G. Samsonidze, A. Gruneis, Y. Miyauchi, S. Maruyama, A. Jorio, G. Dresselhaus, and M. Dresselhaus, "Photoluminescence intensity of single-wall carbon nanotubes," Carbon 44, 873-879 (2006). [doi:10.1016/j.carbon.2005.10.024].

[63] M. Y. Sfeir, T. Beetz, F. Wang, L. Huang, X. Huang, M. Huang, J. Hone, S. O’Brien, J. Misewich, T. Heinz, L. W. Y. Zhu, and L. Brus, "Optical spectroscopy of individual single-walled carbon nanotube of defined chiral structure," Science 312, 554-556 (2006). [doi: $10.1126 /$ science.1124602].

[64] O. V. Kibis, M. R. da Costa, and M. E. Portnoi, "Carbon nanotubes as a basis for novel terahertz devices,” Physica E 40, 1766-1768 (2008). [doi:10.1016/j.physe.2007.11.013].

[65] M. E. Portnoi, O. V. Kibis, and M. R. da Costa, "Terahertz applications of carbon nanotubes,” Superlat. Microst. 43, 399-407 (2008). [doi:10.1016/j.spmi.2007.07.026].

[66] O. V. Kibis, S. V. Malevannyy, L. Hugget, D. G. W. Parf tt, and M. E. Portnoi, "Superlattice properties of helical nanostructures in a transverse electric field," Electromagnetics $\mathbf{2 5}$, 425-435 (2005). [doi:10.1080/02726340590957416].

[67] O. V. Kibis and M. E. Portnoi, "Semiconductor nanohelix in electric field: a superlattice of the new type," Tech. Phys. Lett. 33, 878-880 (2007). [doi:10.1134/S1063785007100215].

[68] O. V. Kibis and M. E. Portnoi, "Superlattice properties of semiconductor nanohelices in a transverse electric field," Physica E 40, 1899-1901 (2008). [doi:10.1016/j.physe.2007.08.054].

[69] O. V. Kibis, D. G. W. Parf tt, and M. Portnoi, "Superlattice properties of carbon nanotubes in a transverse electric field," Phys. Rev. B 71, 035411-035415 (2005) [doi:10.1103/PhysRevB.71.035411]. 
[70] K. N. Alekseev, M. V. Gorkunov, N. V. Demarina, T. Hyart, N. V. Alexeeva, and A. V. Shorokhov, "Suppressed absolute negative conductance and generation of highfrequency radiation in semiconductor superlattices," Europhys. Lett. 73, 934-940 (2006). [doi:10.1209/epl/i2006-10025-9].

[71] H. Ajiki and T. Ando, "Energy bands of carbon nanotubes in magnetic fields," J. Phys. Soc. Jpn. 65, 505-514 (1996). [doi:10.1143/JPSJ.65.505].

[72] O. V. Kibis, "Features of electron-phonon interaction in nanotubes with chiral symmetry placed in a magnetic field," Phys. Sol. State 43, 2336-2343 (2001). [doi:10.1134/1.1427966].

[73] O. V. Kibis, "Electronic phenomena in chiral carbon nanotubes in the presence of a magnetic field," Physica E 12, 741-744 (2002). [doi:10.1016/S1386-9477(01)00466-0].

[74] M. E. Portnoi, M. R. da Costa, O. V. Kibis, and I. A. Shelykh, "Magnetically controlled terahertz absorption and emission in carbon nanotubes," Int. J. Mod. Phys. B 23, 28462850 (2009). [doi:10.1142/S0217979209062438].

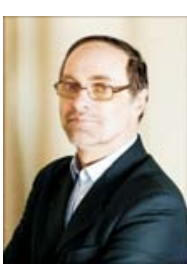

Konstantin G. Batrakov is a senior researcher at the Institute for Nuclear Problems and an associate professor at Belarus State University, Minsk, Belarus. He received his $\mathrm{PhD}$ in Theoretical Physics in 1993 from the Institute for Nuclear Problems, Minsk, Belarus. He has carried out research for more than 20 years. The results, obtained by Dr. Batrakov in co-authorship with his colleagues in the field of Free Electron Lasers, have been used in the first lasing of Volume Free Electron Laser in the Institute for Nuclear Problems. His present research interest is possible nanotubes applications as monomolecular light emitters in the $\mathrm{THz}$ frequency region (nano-scale free electron laser).

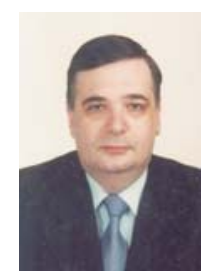

Oleg V. Kibis is a professor in the Department of Applied and Theoretical Physics at the Novosibirsk State Technical University, Russia. He received his $\mathrm{PhD}$ and $\mathrm{ScD}$ degrees in condensed matter physics from Institute of Semiconductor Physics (Siberian Branch of Russian Academy of Sciences, Novosibirsk) in 1988 and 2000, respectively. He is the author or coauthor of more than 100 conference and journal papers. His current research interest is the theoretical physics of different nanostructures, including both semiconductor nanostructures and carbon nanotubes. More details can be obtained from his web-site: www.kibis.ru

Polina P. Kuzhir is a senior researcher at the Institute for Nuclear Problems, Belarus State University, Minsk, Belarus. She received her PhD in High Energy Physics, in 1996 from the Institute of Physics, Belarus Academy of Science, Minsk, Belarus. She carried out research in the field of Particle Physics and Quantum Field Theory for more than 15 years. The results, obtained by Dr Kuzhir in co-authorship with her colleagues from the National Center of Particle and HEP Physics within the limits of Standard Model of Electroweak and Strong interactions have been widely used for the data processing of the world leading experiments on Particle Physics in CERN, SLAC, DESY. Now she is actively involved in the theoretical and experimental research of electromagnetic response of nanocarbon composite materials based on CNT and fullerene-like structures obtained by thermal transformation of nanodiamonds - onion-like carbon. At the same time she contributes to the pioneering investigation of the potential of nanotubes as monomolecular light emitter in $\mathrm{THz}$ frequency region (nano-scaled free electron laser). 


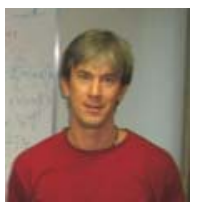

Marcelo Rosenau da Costa is currently a postdoctoral researcher at the International Center for Condensed Matter Physics - ICCMP, Brazil. He received his BS degree in physics from the University Federal de Curitiba, in 1996, and his MS and PhD degrees in physics from the University Estadual de Campinas - UNICAMP, in 1998 and 2004, respectively. He is the author of more than 10 journal papers. His current research interests include quantum point contacts, quantum Hall systems, and carbon nanotubes.

Mikhail E. Portnoi is a Senior Lecturer in Theoretical Physics at the Univer-

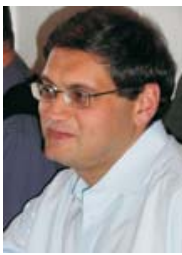
sity of Exeter, UK. He obtained his MSc degree in electrical engineering from the Leningrad Electrotechnical Institute in 1988, his Candidate of PhysicalMathematical Sciences degree from the A.F. Ioffe Physical-Technical Institute in 1994, and $\mathrm{PhD}$ in physics from the University of Utah in 1996. He is the author of more than 60 journal and 80 conference proceedings papers, as well as three book chapters. He is an associate editor of Journal of Nanophotonics. His research interests include carbon nanotubes, graphene, excitons and electron-hole plasma in semiconductor quantum wells, THz optoelectronics, optical spin orientation in nanostructures and the quantum Hall effect. 